\title{
Floquet Solutions for the 1-Dimensional Quasi-Periodic Schrödinger Equation
}

\section{H. Eliasson}

Department of Mathematics, Royal Institute of Technology, S-10044 Stockholm, Sweden

Received June 10, 1991

\begin{abstract}
We show that the 1-dimensional Schrödinger equation with a quasiperiodic potential which is analytic on its hull admits a Floquet representation for almost every energy $E$ in the upper part of the spectrum. We prove that the upper part of the spectrum is purely absolutely continuous and that, for a generic potential, it is a Cantor set. We also show that for a small potential these results extend to the whole spectrum.
\end{abstract}

\section{Introduction}

In this paper we will consider the Schrödinger equation

$$
(\mathscr{L} y)(t)=-y^{\prime \prime}(t)+q(\omega t)=E y(t)
$$

for a real quasi-periodic potential $q(\omega t)$ with frequency vector $\omega$, and for large energies $E$ or small potential $q$. We will study the existence and non-existence of Floquet solutions or Bloch waves, i.e. solutions of the form $y(t)=e^{k t}\left(p_{1}(t)+t p_{2}(t)\right)$, where $k$ is a constant and $p_{1}, p_{2}$ are quasi-periodic functions with the frequency vector $\omega$ or $\frac{\omega}{2}$. We will also study the nature of the spectrum $\sigma(\overline{\mathscr{L}})$, where $\overline{\mathscr{L}}$ is the closure of the operator

$$
\mathscr{L}: C_{c}^{\prime \prime}(\mathbf{R}) \rightarrow L^{2}(\mathbf{R})
$$

in the space $L^{2}(\mathbf{R})$ of complex square integrable functions on $\mathbf{R}$.

We shall assume that $q: \mathbf{T}^{d} \rightarrow \mathbf{R}, \mathbf{T}=\mathbf{R} /(2 \pi \mathbf{Z})$, is analytic in a complex neighbourhood $|\operatorname{Im} x|<r$ of $\mathbf{T}^{d}$, and we shall use the norm

$$
|q|_{r}=\sup _{|\operatorname{Im} x|<r}|q(x)| .
$$

We shall also assume that $\omega$ is diophantine, i.e.

$$
|\langle n\rangle| \geqq|n|^{-\tau}, \quad n \in \mathbf{Z}^{d} \backslash 0
$$


for some $\tau>d-1$, where $\langle n\rangle=\langle n, \omega\rangle$ is the scalar product in $\mathbf{R}^{d}$.

Let $\mathscr{M}=\left\{\frac{\langle n\rangle}{2}: n \in \mathbf{Z}^{d}\right\}$-half the frequency module of $q$-and let $\rho=\rho(E)$ be the rotation number of $(*) . \rho$ is a monotone and continuous function, and

$$
\sigma(\overline{\mathscr{L}})=\overline{\mathbf{R} \backslash \rho^{-1}(\mathscr{M})}
$$

(see [1]). The connected components of int $\left(\rho^{-1}(\mathscr{M})\right)$ are the gaps. So the resolvent set of $\overline{\mathscr{L}}$ is the union of all gaps. A collapsed gap is a point $\{E\}$ for which $\rho(E) \in \mathscr{M}$ and $\rho^{-1}(\rho(E))=\{E\}$.

A real number $\rho$ is said to be diophantine (with respect to $\mathscr{M}$ ) if there exist $K$ and $\sigma$ such that

$$
\left|\rho-\frac{\langle n\rangle}{2}\right| \geqq K^{-1}|n|^{-\sigma}, \quad n \in \mathbf{Z}^{d} \backslash 0,
$$

and it is said to be rational (with respect to $\mathscr{M}$ ) if $\rho \in \mathscr{M}$.

Description of the Results. We shall formulate our result for the matrix equation corresponding to $(*)$.

$$
X^{\prime}(t)=\left(\begin{array}{cc}
0 & 1 \\
q(\omega t)-E & 0
\end{array}\right) X(t)
$$

Theorem A. There exists a constant $\mathbf{C}=\mathbf{C}(\tau, r)$ such that if

$$
E_{0}(s)= \begin{cases}\left(\frac{s}{\mathbf{C}}\right)^{2} & s \geqq \mathbf{C} \\ -\infty & s<\mathbf{C}\end{cases}
$$

then the following hold for $E>E_{0}\left(|q|_{r}\right)$.

A.1. If $\rho(E)$ is diophantine or rational, then there exists a matrix $A=A(E)$ in $\operatorname{sl}(2, \mathbf{R})$ and an analytic matrix valued function $Y: \mathbf{T}^{d} \rightarrow G l(2, \mathbf{R})$, also depending on $E$, such that $X(t)=Y\left(\frac{\omega}{2} t\right) e^{A t}$.

A2. If $\rho(E)$ is neither diophantine nor rational, then

$$
\liminf _{|t| \rightarrow \infty}|X(t)-X(0)|<\frac{1}{2}|X(0)| \text { and } \lim _{|t| \rightarrow \infty} \frac{|X(t)|}{t}=0 .
$$

Theorem $\mathrm{A}$ is a statement about reducibility of equation $(* *)$. Indeed, $Y\left(\frac{\omega}{2} t\right)$ solves the equation

$$
\frac{d}{d t} Y\left(\frac{\omega t}{2}\right)=\left(\begin{array}{cc}
0 & 1 \\
q(\omega t)-E & 0
\end{array}\right) Y\left(\frac{\omega t}{2}\right)-Y\left(\frac{\omega t}{2}\right) A(E)
$$

for almost every rotation $\rho(E)>\rho\left(E_{0}\right)$. Linear periodic systems are always reducible as was shown by Floquet - Floquet theory - but the situation for $\mathrm{q}-\mathrm{p}$ systems is more complicated. 
In the resolvent set it is known that $(* *)$ is reducible (see [2]). The first positive result in the spectrum $\sigma(\overline{\mathscr{L}})$ was obtained by Dinaburg-Sinai [3] (see also [4]). They showed the existence of a set $\left.\mathscr{R}_{1} \subset\right] E_{0}, \infty[\cap \sigma(\overline{\mathscr{L}})$ such that $(* *)$ is reducible and $\rho(E)$ is diophantine for all $E \in \mathscr{R}_{1}$. This set, however, is not of full measure in ]$E_{0}, \infty\left[\cap \sigma(\overline{\mathscr{L}})\right.$. Moser-Pöschel in [2] constructed a set $\left.\mathscr{R}_{2} \subset\right] E_{0}, \infty[\cap \sigma(\overline{\mathscr{L}})$ for which $(* *)$ is reducible and $\rho(E)$ is rational for all $E \in \mathscr{R}_{2}$. But this set was also not as large as one could reasonably hope for.

In fact, both $\mathscr{R}_{1}$ and $\mathscr{R}_{2}$ are defined by certain arithmetric conditions on $\rho(E)$, and these conditions can be relaxed only by letting $E_{0}$ become larger - which essentially amounts to require a stronger smallness condition. The principal achievement of this paper is that the smallness condition is completely freed from any dependence of the arithmetic properties of $\rho(E)$ other than being diophantine or rational.

By Theorem A1, (**) is reducible for a.e. rotation number $\rho \in\left\{\rho(E): E>E_{0}\right\}$, but one may ask if this also holds for a.e. $E>E_{0}$. That this indeed is the case is the content of the following corollary.

Corollary. $2 \rho(E) \rho^{\prime}(E) \geqq 1$ for almost every $\left.E \in \sigma(\overline{\mathscr{L}}) \cap\right] E_{0}, \infty[$. In particular, the set of all $E>E_{0}$ for which $\rho(E)$ is neither diophantine nor rational is of measure 0.

The corollary is almost immediate. It is known that $2 \rho(E) \rho^{\prime}(E) \geqq 1$ for a.e. $E \in\{E: \gamma(E)=0\}$, where $\gamma(E)$ is the "maximal Lyapunov exponent" of $(*)$ (see [5]). If now $\rho(E)$ is diophantine, then $\gamma(E)=0$ by $\mathrm{A} 1$, and if if $\rho(E)$ is neither diophantine nor rational, then $\gamma(E)=0$ by $\mathrm{A} 2$. Hence $\gamma(E)=0$ for a.e. $E$ in the upper part of the spectrum.

One may reasonably ask if $(* *)$ is reducible for all $E>E_{0}$. There are of course $q$ for which this is the case $-q=$ const for example - but this is not the generic situation. In fact, if $X$ is reducible with $\rho(E)$ neither diophantine nor rational, then $\lim _{|t| \rightarrow \infty} \frac{|X(t)|}{t}=0$ by $\mathrm{A} 2$, which implies that $X$ is bounded. The existence of unbounded such solutions is the content of the next theorem.

Theorem B. For $E>E_{0}\left(|q|_{r}\right)$ the following hold.

B1. The matrix $A(E)=0$ if $\{E\}$ is a collapsed gap, and it is nilpotent $\neq 0$ if $E$ is an endpoint of a gap.

B2. For a generic set of $q$ 's, in the $|q|_{r}$-topology, there exist $E>E_{0}\left(|q|_{r}\right)$ for which $X$ is unbounded and $\rho(E)$ is neither diophantine nor rational.

There are several examples in the literature of non-reducible linear q-p systems, but these examples are all non-smooth in the sense that $q$ only is continuous on $\mathbf{T}^{d}$. To our knowledge the only smooth examples are [6] (see also [7]). These examples sit in the bottom of the spectrum and are exponentially localized, while the above result concerns the upper part of the spectrum, and the solutions are clearly not localized because of $\mathrm{A} 2$.

Theorem C. For $E>E_{0}\left(|q|_{r}\right)$ the following hold.

C1. For a generic potential $q$, in the $|q|_{r}$-topology, $\left.\sigma(\overline{\mathscr{L}}) \cap\right] E_{0}\left(|q|_{r}\right), \infty[$ is a Cantor set.

C2. $\sigma(\overline{\mathscr{L}}) \cap] E_{0}\left(|q|_{r}\right), \infty[$ is purely absolutely continuous. In particular, there are no point eigenvalues in $] E_{0}\left(|q|_{r}\right), \infty[$. 
$\mathrm{C} 1$ follows, as described in [2], from Theorem A. And from A it is also clear that there is no point spectrum in $] E_{0}\left(|q|_{r}\right), \infty[$. (For previous results in this direction, see $[8,9]$.) In particular, if $q$ is small there is no point spectrum at all, in distinction to the case when $q$ is large and point spectrum can occur [6].

It was in [3] that the existence of some absolutely continuous spectrum was first proven. From [10] we know that the Lebesgue measure $\left|\sigma_{\mathrm{ac}}(\overline{\mathscr{L}}) \Delta \gamma^{-1}(0)\right|$ is 0 , and from [1] we know that $\gamma^{-1}(0) \subset \sigma(\overline{\mathscr{L}})$. Since $\gamma=0$ on $\sigma(\overline{\mathscr{L}})$ by Theorem A, and since $\sigma_{\text {ac }}(\overline{\mathscr{L}}) \subset \sigma(\overline{\mathscr{L}})$ we have that $\left|\sigma(\overline{\mathscr{L}}) \Delta \sigma_{\text {ac }}(\overline{\mathscr{L}})\right|=0$. Since both $\sigma(\overline{\mathscr{L}})$ and $\sigma_{\text {ac }}(\overline{\mathscr{S}})$ are closed we can conclude that they are equal, if we just know that, for any interval $I$,

$$
\sigma(\overline{\mathscr{L}}) \cap I \neq \varnothing \Rightarrow|\sigma(\overline{\mathscr{L}}) \cap I|>0
$$

This follows quite easily from the estimates given below, but it does not establish $\mathrm{C} 2$, since there may still be some singular continuous spectrum. In order to rule this out we shall show, following [3], that all spectral measures are absolutely continuous with respect to the Lebesgue measure on the set $] E_{0}\left(|q|_{r}\right), \infty[$.

Let us also mention that some cases of the discrete Schrödinger equation has been shown to have purely absolutely continuous spectrum $[11,12]$.

Idea of proof. The problem is to study an equation

$$
X^{\prime}=\left(A_{1}+F_{1}\right) X
$$

where $A_{1}$ is constant and $F_{1}$ is small, $\left|F_{1}\right| \sim \varepsilon_{1}$ say. This is obvious if $q$ is small, but it is true for any $q$, as was found by Dinaburg-Sinai, if $E$ is large enough.

One wants to construct a transformation $Y_{2}$ such that

$$
Y_{2}^{\prime}=\left(A_{1}+F_{1}\right) Y_{2}-Y_{2}\left(A_{2}+F_{2}\right) \text {, }
$$

where $A_{2}$ is constant and $F_{2}$ is much smaller than $F_{1}$, in order to start up an iteration. To do this one solves a linear equation

$$
Y_{2}^{\prime}=\left[A_{1}, Y_{2}\right]+F_{1}-\left(A_{2}-A_{1}\right)
$$

and simply defines $F_{2}$ by (1.1). In order for $F_{2}$ to be small one needs a diophantine condition on the imaginary parts $\pm i \alpha_{1}$ of the eigenvalues of $A_{1}$ :

$$
\left|2 \alpha_{1}-\langle n\rangle\right| \geqq K_{1}^{-1}|n|^{-s}, \quad n \in \mathbf{Z}^{d} \backslash 0,
$$

where $K_{1}$ may be large but not too large. In fact, $K_{1} \varepsilon_{1}$ must be small but may be much larger than $\varepsilon_{1}$, so one can take $K_{1} \sim \varepsilon_{1}^{-\sigma}$ for $\sigma<1$, for example.

If this holds, then one gets a solution of (1.1) with $Y_{2}$ close to the identity and $A_{2}$ close to $A_{1}$. And then one can repeat the same procedure for $A_{2}+F_{2}$ if just

$$
\left|2 \alpha_{2}-\langle n\rangle\right| \geqq K_{2}^{-1}|n|^{-s}, \quad n \in \mathbf{Z}^{d} \backslash 0,
$$

for some $K_{2} \sim\left|F_{2}\right|^{-\sigma}$. This is the approach taken by Dinaburg-Sinai in [3].

One crucial point here is that one tries to construct the transformation $Y_{2}$ as being close to the identity. It is this requirement which imposes the condition (1.3), or at least a part of it, on $\alpha_{1}$. In [2], Moser-Pöschel studied the case where (1.3) is satisfied (for a reasonable $K_{1}$ ) for all $n \in \mathbf{Z}^{d} \backslash 0$ except for one. They found that one could still transform $A_{1}+F_{1}$ to $A_{2}+F_{2}$ with $F_{2}$ small, if one permits a transformation $Y_{2}$ which is close, not to the identity, but to an exponential function $e^{B t}$. (Of course, $Y_{2}$ will not be a solution of the linear equation (1.2) in this case, and $A_{2}$ will not be close to $A_{1}$.) 
So using this idea one can relax the condition on $\alpha_{1}$ in such a way that one requires that the inequality in (1.3) holds for all integer vectors $n \in \mathbf{Z}^{d} \backslash 0$ except possibly one. Of course, even this weaker condition is not always fulfilled, but it is a well known fact that it suffices to require such an inequality for $|n| \leqq N_{1}$, where $N_{1}$ can be taken to be $\sim \log \left(\frac{1}{\varepsilon_{1}}\right)$, since $F_{1}$ is analytic and its Fourier
coefficients decay exponentially.

Hence, we must require that

$$
\left|2 \alpha_{1}-\langle n\rangle\right| \geqq K_{1}^{-1}|n|^{-s}, \quad 0<|n| \leqq N_{1}, \quad \text { except possibly one. }
$$

Now, (1.4) is always fulfilled because if

$$
\left|2 \alpha_{1}-\langle n\rangle\right|<K_{1}^{-1}|n|^{-s} \text { and }\left|2 \alpha_{1}-\langle m\rangle\right|<K_{1}^{-1}|m|^{-s},
$$

with $|n|,|m| \leqq N_{1}$, it follows that

$$
\left(2 N_{1}\right)^{-\tau} \leqq K_{1}^{-1}\left(|n|^{-s}+|m|^{-s}\right) \leqq 2 K_{1}^{-1} .
$$

But since $K_{1} \sim \varepsilon_{1}^{-\sigma}$ and $N_{1} \sim \log \left(\frac{1}{\varepsilon_{1}}\right)$ this is impossible for $\varepsilon_{1}$ small enough.

So this permits us to always solve (1.1). Repeating this procedure gives eventually a product $Y=\prod Y_{j}$ such that

$$
Y^{\prime}=\left(A_{1}+F_{1}\right) Y-Y A,
$$

with $A$ constant. But since $Y_{j}$ is close to an exponential $e^{B_{j} t}$, the convergence of this product is unsure unless $B_{j}=0$ for all $j$ sufficiently large. This is indeed the case if the rotation number $\rho$ is diophantine or rational, and this is the whole proof of A1. Moreover, even if the product does not always converge uniformly on $\mathbf{T}^{d}$, it does converge uniformly on compact intervals in $\mathbf{R}$ and, hence, gives a representation of the solution. This provides the information for proving A2. So the result in A2, we like to stress this, is obtained by a perturbation method which is not absolutely convergent.

The other results will follow by the same approach but will require a more detailed description of $\alpha_{1}$ and its dependence on parameters.

Outline of the Paper. In Sect. 2 we prove a basic small divisor lemma and in Sect. 3 an inductive lemma - these are standard in every KAM-approach. The set-up is chosen with the only aim of getting as simple and uniform estimates as possible. This has of course a price and the smallness condition obtained is therefore not to be taken very seriously. In Sect. 4 we prove A1 and A2 as easy consequences of the inductive lemma.

In Sect. 5 we prove B1. This requires a substantial amount of work, but B1, or rather its "only if" part, is essential also for the proof of B2. The Cantor structure of the spectrum follows from A in the way described in [2], and we only explain this without giving any details. B2 is proven in Sect. 6, and the generic condition is that "all gaps are there." More precisely, we show that on any interval $\Delta \subset] E_{0}, \infty$ [ in which there is a dense set of gaps, there exist solutions as in $\mathrm{B} 2$. The absolute continuity of the upper part of the spectrum is proven in Sects. 7 and 8 following Dinaburg-Sinai.

For the rotation number of $(*)$, or of the matrix solution $X(t)$ of $(* *)$, and its properties we refer to [1]. However, in the proof we must consider rotation numbers 
of other matrices than $X(t)$, so we describe this concept and some elementary properties in an Appendix.

\section{The Small Divisor Lemma}

Let $\mathscr{B}_{r}$ be the space of all analytic functions $F: T^{d} \rightarrow g l(2, \mathbf{C})$ for which

$$
|F|_{r}=\sup _{|\operatorname{Im} x|<r}|F(x)|<\infty .
$$

||$_{r}$ is a norm making $\mathscr{B}_{r}$ into a Banach space. Let $\mathscr{B} \bigcup_{r>0} \mathscr{B}_{r}$.

If $F$ depends on a parameter $\lambda \in \Delta \subset \mathbf{R}$, we say that $F$ is $C^{2}$ in $\lambda$ if $\lambda \rightarrow F_{\lambda} \in \mathscr{B}_{r}$ is $C^{2}$. This is almost equivalent to the requirement that $\lambda \rightarrow F_{\lambda}(x) \in \operatorname{gl}(2, \mathbf{C})$ is $C^{2}$ for each $x$. Clearly the first condition implies the second, and the second implies that $\lambda \rightarrow F_{\lambda} \in \mathscr{B}_{s}$ is $C^{2}$ for each's $s$. We say that $F$ is piecewise $C^{2}$ in $\lambda$ on some set $\Delta \subset \mathbf{R}$ if there exist a finite set $\left\{\lambda_{i}\right\}$ in $\Delta$ such that $F$ is $C^{2}$ on $\Delta \backslash\left\{\lambda_{i}\right\}$ and such that the right and left limits of $\partial^{v} F, v=0,1,2, \partial=\frac{\partial}{\partial \lambda}$, exist at all points $\lambda_{i}$, whenever such a limit makes sense.

For $F \in \mathscr{B}_{r}$ we define

$$
\hat{\sigma}(F)=\left\{n \in \mathbf{Z}^{d}: \hat{F}(n) \neq 0\right\},
$$

where $\hat{F}(n)$ is the $n^{\text {th }}$ Fourier coefficient of $F$.

Lemma 1. Let $A=A(\lambda) \in \operatorname{sl}(2, \mathrm{C})$ have eigenvalues $\pm e(\lambda)$, and assume that $|A(\lambda)-\tilde{\lambda} J|<3$ for some $\tilde{\lambda}=\tilde{\lambda}(\lambda)$. Let $F \in \mathscr{B}_{r}$ and assume

$$
|i\langle n\rangle \pm 2 e(\lambda)| \geqq K^{-1}|n|^{-1}, \quad n \in \hat{\sigma}(F) .
$$

Then there exists a unique $Y \in \mathscr{B}$ such that

$$
\left\{\begin{array}{l}
\partial_{\omega} T=[A, Y]+F \\
\hat{\sigma}(Y) \subset \hat{\sigma}(F),
\end{array}\right.
$$

where $\partial_{\omega}=\langle\nabla, \omega\rangle$.Y satisfies the estimate

$$
|Y|_{s} \leqq c \frac{K^{2}}{(r-s)^{3 \tau}}|F|_{r}, \quad s<r .
$$

Moreover, if $F, A \in \mathscr{B}_{r}$ are $C^{2}$ or pw. $C^{2}$ in $\lambda$, then $Y \in \mathscr{B}_{s}$ also, and

$$
\left\{\begin{aligned}
|\partial Y|_{s} \leqq c & {\left[\frac{K^{2}}{(r-s)^{3 \tau}}|\partial F|_{r}+\left(\frac{K^{2}}{(r-s)^{3 \tau}}\right)^{2}|\partial A||F|_{r}\right] } \\
\left|\partial^{2} Y\right|_{s} \leqq c & {\left[\frac{K^{2}}{(r-s)^{3 \tau}}\left|\partial^{2} F\right|_{r}+\left(\frac{K^{2}}{(r-s)^{3 \tau}}\right)^{2}\left(\left|\partial^{2} A\right||F|_{r}+|\partial A \| \partial F|_{r}\right)\right.} \\
& \left.+\left(\frac{K^{2}}{(r-s)^{3 \tau}}\right)^{3}|\partial A|^{2}|F|_{r}\right]
\end{aligned}\right.
$$

for all $s<r$. The constant $c$ only depends on $\tau$. 
Proof. We write $A=D T D^{*}$ with

$$
T=\left(\begin{array}{cc}
e & p \\
0 & -e
\end{array}\right),
$$

where $D$ is an Hermitian matrix. The condition $|A(\lambda)-\tilde{\lambda} J|<3$ gives a bound for $p$, and that is the only reason for this condition.

Now, putting $Z=D^{*} Y D$ we get

$$
\partial_{\omega} Z-[T, Z]=D^{*} F D=G \text {. }
$$

If

$$
Z=\left(\begin{array}{ll}
u & v \\
w & z
\end{array}\right), \quad G=\left(\begin{array}{ll}
a & b \\
c & d
\end{array}\right),
$$

then we get the equations

$$
\left\{\begin{array}{l}
\partial_{\omega} w+2 e w=c \\
\partial_{\omega} u=a+p w \\
\partial_{\omega} z=d-p w \\
\partial_{\omega} v-2 e v=b-p(u-z) .
\end{array}\right.
$$

These equations can be solved in Fourier series, and this proves the existence of $Z$ and also its uniqueness under the condition $\hat{\sigma}(Z) \subset \hat{\sigma}(G)$.

The estimates are standard and we get

$$
|Z|_{s} \leqq c \frac{K^{2}}{(r-s)^{3 \tau}}|G|_{r}, \quad s<r,
$$

where the constant only depends on $\tau$. (This estimate is far from optimal.) This gives the estimate of $Y$, since $|D| \leqq 1$.

Suppose now that $F$ and $A$ are $C^{2}$ in a neighbourhood of $\lambda$. It is clear from the construction that $Y$ is also $C^{2}$ near $\lambda$ if $e(\lambda) \neq 0$. And the same holds if $F$ and $A$ are $C^{2}$ only in a one-sided neighbourhood of $\lambda$.

If now $e(\lambda)=0$ and $A$ and $F$ are $C^{1}$ at $\lambda$, then

$$
\begin{gathered}
\partial_{\omega}(Y(\cdot, \lambda+\varepsilon)-Y(\cdot, \lambda))-[(A(\lambda+\varepsilon), Y(\cdot, \lambda+\varepsilon)-Y(\cdot, \lambda)] \\
\quad=F(\cdot, \lambda+\varepsilon)-F(\cdot, \lambda)+[A(\cdot, \lambda+\varepsilon)-A(\cdot, \lambda), Y(\lambda)] .
\end{gathered}
$$

Using the estimates of the solution of this equation we get that $Y$ is differentiable at $\lambda$. The other cases are treated in the same way, and this shows that if $F$ and $A$ are $C^{2}$ or pw. $C^{2}$ in $\lambda$ then $Y \in \mathscr{B}_{s}$ also. Now, differentiating the equation for $Y$ gives

$$
\begin{aligned}
\partial_{\omega}(\partial Y)-[A, \partial Y] & =\partial F+[\partial A, Y], \\
\partial_{\omega}\left(\partial^{2} Y\right)-\left[A, \partial^{2} Y\right] & =\partial^{2} F+\left[\partial^{2} A, Y\right]+2[\partial A, \partial Y],
\end{aligned}
$$

from which we easily deduce the estimates.

\section{The Inductive Lemma}

Let $A \in \operatorname{sl}(2, \mathbf{R})$. Since $\operatorname{tr} A=0$, its two eigenvalues coincide up to a sign, and since $A$ is real they are either both real or both purely imaginary. Hence, the imaginary 
parts of the eigenvalues are $= \pm i \alpha$. Of course, $\alpha$ is defined only up to a sign, but we shall require that

$$
A=\alpha M J M^{-1}, \quad J=\left(\begin{array}{cc}
0 & 1 \\
-1 & 0
\end{array}\right)
$$

for some real matrix $M$ with $\operatorname{det} M>0$. This defines $\alpha$ uniquely, and we shall call it the rotation number of $A$ (it is the rotation number of $t \rightarrow e^{t A}$, see Appendix.) If $A=A(\lambda)$ is continuous in $\lambda$, then $\alpha(\lambda)$ is also continuous in $\lambda$, and if $A=A(\lambda)$ is pw. $C^{2}$ on $\Delta$, then $\alpha(\lambda)$ is pw. continuous on $\Delta$ and pw. $C^{2}$ on $\Delta \backslash \alpha^{-1}(0)$.

Let $\left\{r_{j}\right\}$ be a decreasing sequence of positive numbers such that $r_{j}-r_{j+1} \geqq 2^{-j} \frac{r_{1}}{2}$ for each $j$. Let $\varepsilon_{j+1}=\varepsilon_{j}^{1+\sigma}$, where $0<\sigma<1$, and let

$$
N_{j}=\frac{2 \sigma}{r_{j}-r_{j+1}} \log \left(\frac{1}{\varepsilon_{j}}\right)
$$

for each $j$. It follows that

$$
\varepsilon_{j}^{\sigma} \leqq\left(\frac{4 \sigma}{r_{1}(1+\sigma)} \log \left(\frac{1}{\varepsilon_{1}}\right)(2+2 \sigma)^{j}\right)^{-4 \tau} \leqq N_{j}^{-4 \tau}
$$

for all $j$, if just $\varepsilon_{1}$ is small enough.

For example, it suffices that

$$
\varepsilon_{1} \leqq c r_{1}^{((4 \tau / \sigma)+1)}
$$

for some all constant $c$ that only depends on $\tau$ and $\sigma$. All constants in this paper will be denoted by $c$. They will only depend on $\sigma$ and $\tau$, unless explicitly stated otherwise. $\sigma$ is a fixed small number, for example $\sigma \leqq \frac{1}{33}$ will satisfy all our needs.

Let now $A_{1}(\lambda) \in \operatorname{sl}(2, \mathbf{R})$ and $F_{1}(\cdot, \lambda) \in \mathscr{B}_{r}$ be real and pw. $C^{2}$ in $\lambda \in \Delta$. We assume that $\operatorname{tr}\left\langle F_{1}\right\rangle=0$, where \langle\rangle denotes the mean value over $\mathbf{T}^{d}$ and we also assume

$$
\begin{cases}\left|A_{1}(\lambda)-\tilde{\lambda}_{1}(\lambda) J\right|<2, & \text { some } \tilde{\lambda}_{1}(\lambda) \\ \mid \partial^{v}\left(A_{1}(\lambda) \mid<\varepsilon_{1}^{-v \sigma},\right. & v=1,2, \\ \left|\partial^{v} F_{1}\right|_{r_{1}}<\varepsilon_{1}, & v=0,1,2\end{cases}
$$

where, we recall, $\partial=\frac{\partial}{\partial \lambda}$. Then we have the following lemma.

Lemma 2. There exists a constant $C=C(\tau, \sigma)$ such that if $\varepsilon_{1}<C r_{1}^{(4 \tau / \sigma)+1)}$ then, for all $j \geqq 1$, there exist $F_{j+1} \in \mathscr{B}, Y_{j+1}$ and $A_{j+1} \in \operatorname{sl}(2, \mathbf{R})$, all real and pw. $C^{2}$ in $\lambda$ and with $\operatorname{tr}\left\langle F_{j+1}\right\rangle=0$, verifying

$$
\left\langle Y_{j+1}^{\prime}\left(\frac{x}{2}\right), \frac{\omega}{2}\right\rangle=\left(A_{j}+F_{j}(x)\right) Y_{j+1}\left(\frac{x}{2}\right)-Y_{j+1}\left(\frac{x}{2}\right)\left(A_{j+1}+F_{j+1}(x)\right) .
$$

Let $\alpha_{j}$ be the rotation number of $A_{j}$ and let $\Lambda_{j}(m)$ be a finite union of intervals such that

$$
\left\{\lambda:\left|2 \alpha_{j}(\lambda)-\langle m\rangle\right|<\varepsilon_{j}^{\sigma}\right\} \subset \Lambda_{j}(m) \subset\left\{\lambda:\left|2 \alpha_{j}(\lambda)-\langle m\rangle\right|<2 \varepsilon_{j}^{\sigma}\right\}
$$


for $0<|m| \leqq N_{j}$ (see remark below). Let also

$$
\Lambda_{j}(0)=\Delta \backslash \cup \Lambda_{j}(m),
$$

and let $r_{j}-r_{j+1}=\frac{1}{2} r_{j}$ if $\lambda \notin \Lambda_{j}(0)$ and $r_{j}-r_{j+1}=2^{-j} \frac{r_{1}}{2}$ if $\lambda \in \Lambda_{j}(0)$. Then we have the following estimates:

$$
\begin{gathered}
\left|\partial^{v}\left(Y_{j+1}(\cdot, \lambda)-\exp \left\{\frac{\langle m, \cdot\rangle}{\alpha_{j}(\lambda)} A_{j}(\lambda)\right\}\right)\right|_{r_{j+1}}<\varepsilon_{j}^{1 / 2}, \quad v=0,1,2, \quad \lambda \in \Lambda_{j}(m) ; \quad(3.1)_{j+1} \\
\left|\partial^{v} F_{j+1}\right|_{r_{j+1}}<\varepsilon_{j+1}, \quad v=0,1,2 ; \\
\left|\partial^{v}\left(A_{j+1}(\lambda)-\left(1-\frac{\langle m\rangle}{2 \alpha_{j}(\lambda)}\right) A_{j}(\lambda)\right)\right|<\varepsilon_{j+1}^{2 / 3}, \quad v=0,1,2, \quad \lambda \in \Lambda_{j}(m) ; \quad(3.3)_{j+1} \\
\begin{cases}\left|\partial^{v} A_{j+1}\right|<\varepsilon_{j+1}^{-v \sigma}, & v=1,2 \\
\left|A_{j+1}(\lambda)\right|<32\left|\alpha_{j+1}(\lambda)\right| N_{j+1}^{\tau} & \text { if }\left|\alpha_{j+1}(\lambda)\right| \geqq \frac{1}{4} N_{j+1}^{-\tau} .\end{cases}
\end{gathered}
$$

Remark 1. The crucial point in the proof is that the sets $\Lambda_{j}(m), 0<|m| \leqq N_{j}$ are disjoint. In fact, for each $\lambda$ there are infinitely many bad numbers $\frac{\langle n\rangle}{2}$ in $\mathscr{M}$, but there is at most one in the ball $|n| \leqq N_{j}$. So there is at most one bad number which must be taken care of at each step of the iteration.

Remark 2. The components of $\Lambda_{j}(m)$ may be closed, open on half open. For the proof of the lemma we don't need to specify them more than by the two inclusions above. For the proof of Theorem A this is all that is needed, but for the proof of Theorem $\mathrm{B}$ and $\mathrm{C}$ it will be convenient to specify them somewhat more.

Proof of Lemma 2. So we assume that $A_{j}$ and $F_{j}$ satisfy $(3.2-4)_{j}$, and we prove the existence of $Y_{j+1}, F_{j+1}$ and $A_{j+1}$ with the required properties. We must handle the two cases $\lambda \in \Lambda_{j}(0)$ and $\lambda \notin \Lambda_{j}(0)$ separately. But first we shall make sure that $A_{1}$ satisfies (3.4) $)_{1}$. The fact that (3.3) makes no sense will be of no importance.

Clearly the first estimate of (3.4) $)_{1}$ is fulfilled by assumption, and the second is trivial unless $\left|A_{1}\right| \geqq 8$. But this implies that $\left|\tilde{\lambda}_{1}\right|>6$, since $\left|A_{1}(\lambda)-\tilde{\lambda}_{1} J\right|<2$, and hence $\left|\alpha_{1}\right|>\left|\tilde{\lambda}_{1}\right|-2$. Now this gives immediately $\left|A_{1}\right|<2\left|\alpha_{1}\right|$.

Case 1. Suppose $\lambda \in \Lambda_{j}(0)$. Then

$$
\left|2 \alpha_{j}(\lambda)-\langle n\rangle\right| \geqq \varepsilon_{j}^{\sigma}, \quad 0<|n| \leqq N_{j} .
$$

Moreover, by $(3.3)_{k}$ and $(3.4)_{k}, k \leqq j$, we have $\left|A_{j}(\lambda)-\tilde{\lambda}_{j} J\right|<3$ for $\tilde{\lambda}_{j}=0$ or $\tilde{\lambda}_{1}$. (This also holds for $A_{1}$, by assumption.) In order to see this we observe that if $\lambda \in \bigcap_{j}^{j-1} \Lambda_{k}(0)$, then $\left|A_{j}(\lambda)-\tilde{\lambda}_{1} J\right|<2+\varepsilon_{1}^{2 / 3}+\cdots+\varepsilon_{j-1}^{2 / 3}<3$. On the other hand, if $\lambda \in \bigcap_{k+1}^{j-1} \Lambda_{l}(0) \cap \Lambda_{k}(m), m \neq 0$, then

$$
\begin{aligned}
\left|A_{j}(\lambda)\right| & <\varepsilon_{k}^{3 / 2}+\cdots+\varepsilon_{j-1}^{2 / 3}+\left|\left(1-\frac{\langle m\rangle}{2 \alpha_{k}}\right) A_{k}\right|<2 \varepsilon_{k}^{2 / 3}+2 \varepsilon_{k}^{\sigma}\left|\frac{A_{k}}{2 \alpha_{k}}\right| \\
& <2 \varepsilon_{k}^{2 / 3}+\varepsilon_{k}^{\sigma} 32 N_{k}^{\tau}<34 N_{k}^{\tau} \varepsilon_{k}^{\sigma},
\end{aligned}
$$


by (3.4), because $\lambda \in \Lambda_{k}(m)$ implies that $\left|2 \alpha_{k}\right| \geqq N_{k}^{-\tau}-2 \varepsilon_{k}^{\sigma} \geqq \frac{1}{2} N_{k}^{-\tau}$, if $m \neq 0$. (Notice this lower bound on $\alpha_{k}$ which we have on $\Lambda_{k}(m)$ when $m \neq 0$.)

We let now $G(x)$ be the truncated Fourier series

$$
\sum_{0<|n| \leqq N_{j}} \hat{F}_{j}(n) e^{i\langle n, x\rangle}
$$

and we define $Y$ as the solution of

$$
\partial_{\omega} Y-\left[A_{j}, Y\right]=G, \quad \hat{\sigma}(Y) \subset \hat{\sigma}(G),
$$

which exists uniquely by Lemma 1 . Then we define

$$
\left\{\begin{array}{l}
Y_{j+1}(x)=I+Y(2 x) \\
A_{j+1}=A_{j}+\hat{F}_{j}(0) \\
F_{j+1}=(I+Y)^{-1}\left[F_{j} Y-Y \hat{F}_{j}(0)+\left(F_{j}-G-\hat{F}_{j}(0)\right)\right]
\end{array}\right.
$$

These matrices are pw $C^{2}$ in $\lambda \in \Lambda_{j}(0)$ and $\operatorname{tr} A_{j+1}=0$. Moreover, they satisfy the equation, so

$$
\begin{aligned}
\operatorname{tr}\left\langle F_{j+1}\right\rangle & =\left\langle\operatorname{tr} F_{j+1}\right\rangle=\left\langle\operatorname{tr} Y_{j+1}^{-1}\left(A_{j}+F_{j}\right) Y_{j+1}\right\rangle-\left\langle\operatorname{tr} Y_{j+1}^{-1} \partial_{\omega} Y\right\rangle \\
& =-\left\langle\operatorname{tr}\left(\sum(-1)^{k} Y^{k}\right) \partial_{\omega} Y\right\rangle=-\sum(-1)^{k} \frac{1}{k+1} \operatorname{tr}\left\langle\partial_{\omega}\left(Y^{k+1}\right)\right\rangle=0 .
\end{aligned}
$$

So we only need to consider the estimates.

Estimates. We have

$$
\left|\partial^{v} G\right|_{s}<c \varepsilon_{j} N_{j}^{\tau+1}, \quad s=r_{j+1}+\frac{r_{j}-r_{j+1}}{2} .
$$

Then by Lemma 1 and $(3.4)_{j}$ we get

$$
\left|\partial^{v} Y\right|_{r_{j+1}}<c \varepsilon_{j}^{1-(2+3 v) \sigma} N_{j}^{\tau(1+(v+1) 3)+1}, \quad v=0,1,2
$$

and this gives $(3.1)_{j+1}$.

In order to prove $(3.2)_{j+1}$ we first observe that

$$
\left|\partial^{v}\left(F_{j}-G-\hat{F}_{j}(0)\right)\right|_{r_{j+1}}<c \varepsilon_{j}^{1+2 \sigma} N_{j}^{\tau+1}
$$

and that

$$
\left|(I+Y)^{-1}\right|_{r_{j+1}}<2
$$

This gives immediately

$$
\left|F_{j+1}\right|_{r_{j+1}}<c\left(\varepsilon_{j}^{1+2 \sigma} N_{j}^{\tau+1}+N_{j}^{4 \tau+1} \varepsilon_{j}^{2-2 \sigma}\right) .
$$

If we differentiate the expression for $F_{j+1}$ we get

$$
\partial F_{j+1}=-(I+Y)^{-1} \partial Y F_{j+1}+(I+Y)^{-1} \partial\left(F_{j} Y-Y \hat{F}_{j}(0)-\left(F_{j}-G-\hat{F}_{j}(0)\right)\right)
$$

from which we get

$$
\left|\partial F_{j+1}\right|_{r_{j+1}}<c\left(\varepsilon_{j}^{1+2 \sigma} N_{j}^{\tau+1}+N_{j}^{7 \tau+1} \varepsilon_{j}^{2-5 \sigma}\right) .
$$

And by a second differentiation,

$$
\left|\partial^{2} F_{j+1}\right|_{r_{j+1}}<c\left(\varepsilon_{j}^{1+2 \sigma} N_{j}^{\tau+1}+N_{j}^{10 \tau+1} \varepsilon_{j}^{2-8 \sigma}\right) .
$$

(In all these estimates $c$ is a constant that only depends on $\sigma$ and $\tau$.) 
The estimates $(3.3)_{j+1}$ are trivial, as well as the first estimate of $(3.4)_{j+1}$.

The second estimate of $(3.4)_{j+1}$ is non-trivial only if $\left|A_{j+1}(\lambda)\right| \geqq 8$ which implies that $\lambda \in \bigcap_{k=1}^{j} \Lambda_{k}(0)$, i.e.

$$
\left|A_{j+1}(\lambda)-\tilde{\lambda}_{1} J\right|<2+2 \varepsilon_{1}^{2 / 3}<\frac{5}{2} .
$$

Hence $\left|\tilde{\lambda}_{1}\right|>5$. But then $\left|\alpha_{j+1}(\lambda)\right| \geqq\left|\tilde{\lambda}_{1}\right|-\frac{5}{2}>\frac{\left|\tilde{\lambda}_{1}\right|}{2}$. Hence

$$
\left|\frac{A_{j+1}(\lambda)}{\alpha_{j+1}(\lambda)}\right| \leqq 2+\frac{5}{\left|\tilde{\lambda}_{1}\right|} \leqq 3 .
$$

This proves the second estimate of $(3.4)_{j+1}$ in case 1 .

Case 2. Suppose now $\lambda \in \Lambda_{j}(m), m \neq 0$, and let

$$
Z(x)=\exp \left\{\frac{\langle m, x\rangle}{2 \alpha_{j}(\lambda)} A_{j}(\lambda)\right\} .
$$

Then

$$
\partial_{\omega} Z=\left(A_{j}+F_{j}\right) Z-Z\left(B_{j}+G_{j}\right)
$$

where

$$
B_{j}=\left(1-\frac{\langle m\rangle}{2 \alpha_{j}}\right) A_{j}, \quad G_{j}=Z^{-1} F_{j} Z .
$$

(Notice that $G_{j}$ is defined on $\mathbf{T}^{d}$, even if $Z$ is defined only on $(2 \mathbf{T})^{d}$.)

The rotation number of $B_{j}$ is $\beta_{j}=\alpha_{j}-\frac{\langle m\rangle}{2}$, and it satisfies

$$
\left|2 \beta_{j}-\langle n\rangle\right| \geqq\left(5 N_{j}\right)^{-\tau}-2 \varepsilon_{j}^{\sigma} \geqq 2 \varepsilon_{j}^{\sigma}, \quad 0<|n| \leqq 5 N_{j} .
$$

Moreover, by $(3.4)_{j}$ we have

$$
\left|B_{j}\right|<2 \varepsilon_{j}^{\sigma}\left|\frac{A_{j}}{2 \alpha_{j}}\right|<1 .
$$

Let now $G$ be the truncated Fourier series

$$
\sum_{0<|n| \leqq 5 N_{j}} \hat{G}_{j}(n) e^{i\langle n, x\rangle} .
$$

(This truncation is not the same as in case 1.) Then we let $Y$ be the unique solution of

$$
\partial_{\omega} Y-\left[B_{j}, Y\right]=G, \quad \hat{\sigma}(Y) \subset \hat{\sigma}(G)
$$

and we define

$$
\left\{\begin{array}{l}
Y_{j+1}(x)=Z(2 x)(I+Y(2 x)) \\
A_{j+1}=B_{j}+\hat{G}_{j}(0) \\
F_{j+1}=(I+Y)^{-1}\left[G_{j} Y-Y \hat{G}_{j}(0)+\left(G_{j}-G-\hat{G}_{j}(0)\right)\right] .
\end{array}\right.
$$

As in Case 1 all requirements are fulfilled and we only need to consider the estimates. 
Estimates. Notice that

$$
Z(x)=\cos \left(\frac{\langle m, x\rangle}{2}\right) I+\sin \left(\frac{\langle m, x\rangle}{2}\right) \frac{A_{j}}{\alpha_{j}} .
$$

From $(3.4)_{j}$ we get

$$
\left|\partial^{v} Z\right|_{r_{j}}<c N_{j}^{(1+2 v) \tau} \varepsilon_{j}^{-(2+v) \sigma}
$$

since $r_{j+1}=\frac{1}{2} r_{j}$. (Here we have used that on $\Lambda_{j}(m)$ we have a lower bound on $\alpha_{j}$, which gives us upper bounds on the derivatives $\partial \alpha_{j}$ and $\partial^{2} \alpha_{j}$, since

$$
\left.\partial \alpha_{j}=\frac{\partial\left(\operatorname{det} A_{j}\right)}{2 \alpha_{j}} .\right)
$$

The same estimate holds for $Z^{-1}$.

This implies that

$$
\left|\partial^{v} G_{j}\right|_{r_{j}}<c N_{j}^{2(1+v) \tau} \varepsilon_{j}^{1-(4+v) \sigma} .
$$

Moreover, by $(3.4)_{j}$ we get

$$
\left|\partial^{v} B_{j}\right|<c N_{j}^{2 v \tau} \varepsilon_{j}^{-v \sigma} \quad v=1,2 .
$$

Since

$$
\left|\partial^{v} G\right|_{s}<c N_{j}^{r+1}\left|\partial^{v} G_{j}\right|_{r_{j}}, \quad s=r_{j+1}+\frac{r_{j}-r_{j+1}}{2}
$$

we get by Lemma 1 ,

$$
\left|\partial^{v} Y\right|_{r_{j+1}}<c N_{j}^{(6+5 v) \tau+1} \varepsilon_{j}^{1-(6+3 v) \sigma}
$$

which gives $(3.1)_{j+1}$.

In order to prove $(3.2)_{j+1}$, we first observe that

$$
\left|\partial^{v}\left(G_{j}-G-\hat{G}_{j}(0)\right)\right|_{r_{j+1}}<c N_{j}^{(3+2 v) \tau+1} \varepsilon_{j}^{1+(10-4-v) \sigma},
$$

and that

$$
\left|(I+Y)^{-1}\right|_{r_{j+1}}<2
$$

This implies that

$$
\left|\partial^{v} F_{j+1}\right|_{r_{j+1}}<c\left(N_{j}^{(8+5 v) \tau+1} \varepsilon_{j}^{2-(10+3 v) \sigma}+N_{j}^{(3+2 v) \tau+1} \varepsilon_{j}^{1+(10-4-v) \sigma}\right),
$$

which gives $(3.2)_{j+1}$.

(3.3) $)_{j+1}$ is obvious from the estimate of $\partial^{v} G_{j}$ above.

In order to prove $(3.4)_{j+1}$ we observe that

$$
\left|A_{j+1}\right|<2 \varepsilon_{j}^{\sigma}\left|\frac{A_{j}}{2 \alpha_{j}}\right|<1,
$$

so the second estimate is trivial. The only thing that is left is the estimate of $\partial^{v} A_{j+1}$.

We have

$$
\left|\partial^{v} A_{j+1}\right|<\left|\partial^{v} G_{j}\right|_{r_{j}}+\left|\partial^{v} B_{j}\right|
$$


Moreover, we can sharpen the above estimate for $\partial^{v} B_{j}$ :

$$
\left|\partial B_{j}\right|<c_{1} N_{j}^{2 \tau}\left|\partial A_{j}\right|<c_{2} N_{j}^{2 \tau}\left(\varepsilon_{k+1}^{2 / 3}+\left|\partial A_{k+1}\right|\right)<c_{3} N_{j}^{2 \tau} \varepsilon_{k+1}^{-\sigma},
$$

where $k<j$ is such that $\lambda \notin \Lambda_{k}(0)$ and $\lambda \in \Lambda_{l}(0), k<l<j$. And in the same way it follows that

$$
\left|\partial^{2} B_{j}\right|<c N_{j}^{4 \tau} \varepsilon_{k+1}^{-2 \sigma} .
$$

In order to conclude, we must show that $c N_{j}^{2 v \tau} \varepsilon_{k+1}^{-v \sigma}<\varepsilon_{j+1}^{-v \sigma}$ for $v=1,2$. We have shown in (3.5) that $\left|A_{j}(\lambda)\right|<34 N_{k}^{\tau} \varepsilon_{k}^{\sigma}$. On the other hand

$$
N_{j}^{-\tau} \leqq|\langle m\rangle| \leqq\left|\langle m\rangle-2 \alpha_{j}\right|+2\left|\alpha_{j}\right|<2 \varepsilon_{j}^{\sigma}+2\left|A_{j}\right|<70 N_{k}^{\tau} \varepsilon_{k}^{\sigma}
$$

hence,

$$
\varepsilon_{j}^{\sigma / 4} \leqq 70 \varepsilon_{k}^{3 \sigma / 4}
$$

This gives the required estimate and finishes the proof of Lemma 2 in Case 2.

\section{Floquet Solutions - Theorem A}

We now come to the conclusions of Lemma 2. The conclusions will depend on $\lambda$. Each $\lambda$ belongs to a unique set $\cap \Lambda_{j}\left(m_{j}\right), 0 \leqq\left|m_{j}\right| \leqq N_{j}$, which may be void. (Formula (3.6) give a necessary condition for this set to be non-void.) It is clear that for all $\lambda$

$$
\left\{\begin{array}{l}
r_{j} \rightarrow r_{0} \geqq 0 \\
\left|F_{j}\right|_{r_{j}} \rightarrow 0 \\
A_{j} \rightarrow A \text { pointwise }
\end{array}\right.
$$

as $j \rightarrow \infty$.

Suppose now $\lambda$ is such that $m_{j}=0$ for all $j$ sufficiently large. Only for such $\lambda$ can we conclude that

$$
\prod_{2}^{n} Y_{j} \rightarrow Y
$$

in ||$_{r_{0}}, r_{0}>0$. For $\lambda$ not of this type the convergence is unsure and, in fact, often untrue. However, we clearly have $\left|Y_{j}(0)-I\right|<\varepsilon_{j}^{1 / 2}$ for all $j$, so $\prod Y_{j}(0)$ is convergent.

But more is true. For each $\lambda$ the product $\prod Y_{j}\left(\frac{\omega}{2} t\right)$ converges uniformly on compact intervals in $\mathbf{R}$. In order to see this we only need to note that

$$
\left|Y_{j+1}\left(\frac{\omega}{2} t\right)-I\right| \leqq \varepsilon_{j}^{1 / 2}+\left|Z_{j}(\omega t)-I\right|
$$

where

$$
Z_{j}(\omega t)=\exp \left\{\frac{\left\langle m_{j}\right\rangle}{2 \alpha_{j}} A_{j} t\right\}=\cos \left(\frac{\left\langle m_{j}\right\rangle}{2} t\right) I+\sin \left(\frac{\left\langle m_{j}\right\rangle}{2} t\right) \frac{A_{j}}{\alpha_{j}} .
$$

If $m_{j}=0$ then we are done for all $t$, and if $m_{j} \neq 0$ then $\left|2 \alpha_{j}-\left\langle m_{j}\right\rangle\right|<2 \varepsilon_{j}^{\sigma}$, which 


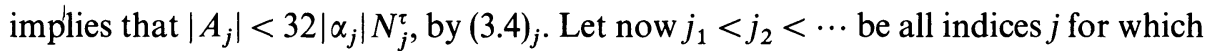
$m_{j} \neq 0$. Then

$$
\left|\left\langle m_{j_{k+1}}\right\rangle\right|<70 N_{j_{k}}^{\tau} \varepsilon_{j_{k}}^{\sigma} \cdot k \geqq 1
$$

(formula (3.6)). Hence, for $t$ with $t N_{j_{k}}^{\tau} \varepsilon_{j_{k}}^{\sigma}$ small, we get by (3.5),

$\left|Z_{j_{k+1}}(\omega t)-I\right| \leqq\left|\left\langle m_{j_{k+1}}\right\rangle t\right|^{2}+\left|\frac{\left\langle m_{j_{k+1}}\right\rangle}{2 \alpha_{j_{k+1}}} t A_{j_{k+1}}\right|<t N_{j_{k}}^{\tau} \varepsilon_{j_{k}}^{\sigma}+2 t 34 N_{j_{k}}^{\tau} \varepsilon_{j_{k}}^{\sigma}<70 t N_{j_{k}}^{\tau} \varepsilon_{j_{k}}^{\sigma}$,

which shows that the product converges uniformly for $t$ bounded.

If now $X_{1}$ is a solution of

$$
X_{1}^{\prime}(t)=\left(A_{1}+F_{1}(\omega t)\right) X(t)
$$

then, by Lemma 2 , we have a representation

$$
X_{1}(t)=Y\left(\frac{\omega}{2} t\right) e^{A t}
$$

Moreover, if $X_{1}$ has a rotation number $\tilde{\rho}(\lambda)$, then we conclude from this representation that

$$
\tilde{\rho}(\lambda)=\frac{1}{2} \sum_{j=1}^{\infty}\left\langle m_{j}\right\rangle+\alpha(\lambda), \quad \lambda \in \cap \Lambda_{j}\left(m_{j}\right)
$$

where $\alpha(\lambda)=\lim \alpha_{j}(\lambda)$. (See the Appendix.)

In order to prove A1 we need a lemma. Let

$$
\rho_{j+1}=\alpha_{j+1}+\frac{1}{2} \sum_{k=1}^{j}\left\langle m_{k}\right\rangle, \quad \lambda \in \bigcap_{k=1}^{j} \Lambda_{k}\left(m_{k}\right),
$$

the point with this definition is that the sequence $\left\{\rho_{j}\right\}$ converges uniformly in $\lambda$.

Lemma 3. a) $\left|\rho_{j+1}-\rho_{j}\right|<c \varepsilon_{j}^{1 / 4}$ for all $j$. In particular, the sequence $\left\{\rho_{j}\right\}$ converges uniformly to the limit $\tilde{\rho}$.

b) If $\lambda \in \bigcap_{1}^{\infty} \Lambda_{j}\left(m_{j}\right)$ and $\tilde{\rho}(\lambda)$ is diophantine or rational, then $m_{j}=0$ for all $j$ sufficiently large.

Proof. For a) it suffices to show that

$$
\left|\alpha_{j+1}(\lambda)-\left(\alpha_{j}(\lambda)-\frac{\langle m\rangle}{2}\right)\right|<c \varepsilon_{j}^{1 / 4}
$$

for $\lambda \in \Lambda_{j}(m)$, which follows from $(3.3)_{j+1}$ by an explicit computation.

For b) suppose

$$
|2 \tilde{\rho}(\lambda)-\langle n\rangle| \geqq K^{-1}|n|^{-s}, \quad|n| \geqq N,
$$

for some $N, K$, as $s>0$. Suppose that there exist $j_{k}$ arbitrarily large such that $m_{j_{k}} \neq 0$. Hence,

$$
\left|2 \alpha_{j_{k}}(\lambda)-\left\langle m_{j_{k}}\right\rangle\right|<2 \varepsilon_{j_{k}}^{\sigma} .
$$


Then

$$
\left|2 \tilde{\rho}(\lambda)-\sum_{1}^{j_{k}}\left\langle m_{l}\right\rangle\right| \leqq 2\left|\tilde{\rho}(\lambda)-\rho_{j_{k}}(\lambda)\right|+\left|2 \alpha_{j_{k}}(\lambda)-\left\langle m_{j_{k}}\right\rangle\right|<4 \varepsilon_{j_{k}}^{\sigma} .
$$

On the other hand

$$
\left|\tilde{\rho}(\lambda)-\sum_{1}^{j_{k}}\left\langle m_{l}\right\rangle\right| \geqq K^{-1}\left(N_{j_{1}}+\cdots+N_{j_{k}}\right)^{-s} \geqq c K^{-1} N_{j_{k}}^{-2 s},
$$

because $N_{j_{l}}^{3}<c N_{j_{l+1}}$ by (3.6). Now this implies that

$$
\varepsilon_{j_{k}}^{-\sigma} \leqq \text { const. }\left(\log \left(\frac{1}{\varepsilon_{1}}\right)(2+2 \sigma)^{j_{k}}\right)^{2 s}
$$

for finitely many $k: s$ which is clearly impossible.

Proof of A1-large energy. Let $E_{0}(s)$ be defined as in the theorem, with $\mathbf{C}(\tau, r)=C(\tau, \sigma) r^{((4 \tau / \sigma)+1)}$ for $\sigma=\frac{1}{33}$ say, where $C$ is the constant of Lemma 2. Let $|q|_{r}$ be arbitrary, and let $E>E_{0}\left(|q|_{r}\right)$. Let $X$ be a solution of $(* *)$. The equation $(* *)$ gets transformed to

$$
X_{1}^{\prime}(t)=\left[A_{1}(\sqrt{E})+F_{1}(\omega t, \sqrt{E})\right] X_{1}(t),
$$

where $A_{1}(\sqrt{E})=\sqrt{E} J$ and

$$
F_{1}(x, \sqrt{E})=\frac{q(x)}{2 \sqrt{E}}\left(\begin{array}{ll}
-1 & -1 \\
+1 & +1
\end{array}\right)
$$

through the change of variables $X_{1}=Y_{1}^{-1} X$, where

$$
Y_{1}=\left(\begin{array}{cc}
1 & 1 \\
-\sqrt{E} & \sqrt{E}
\end{array}\right) .
$$

$X_{1}$ has rotation number $\tilde{\rho}(\sqrt{E})=\rho(E)$ and

$$
\left|\left(\frac{\partial}{\partial \sqrt{E}}\right)^{v} F_{1}\right|_{r}<\varepsilon_{1}=C(\tau, \sigma) r^{(4 \tau / \sigma)+1)}, \quad v=0,1,2,
$$

so it follows from Lemma 2 that the solution $X$ of $(* *)$ has a representation $\tilde{Y}\left(\frac{\omega}{2} t\right) e^{A t}$, where

$$
\tilde{Y}\left(\frac{\omega}{2} t\right)=Y_{1} Y\left(\frac{\omega}{2} t\right) .
$$

Moreover, it follows from Lemma 3 that the product $Y=\prod Y_{j}$ converges uniformly in a complex neighbourhood of $\mathbf{T}^{d}$, if $\rho(E)$ is rational or diophantine.

Proof of A1 - small potential. Let $E_{0}$ and $\mathbf{C}$ be as above. Suppose $|q|_{r}<\mathbf{C}(r, \tau)$ and let $E \in]-1,+1[$. Now $(* *)$ can be written

$$
X_{1}^{\prime}(t)=\left(A_{1}(E)+F_{1}(\omega t, E)\right) X_{1}(t),
$$


where

$$
A_{1}=\left(\begin{array}{cc}
0 & 1 \\
-E & 0
\end{array}\right), \quad F_{1}(x)=\left(\begin{array}{cc}
0 & 0 \\
q(x) & 0
\end{array}\right) .
$$

$X_{1}$ has rotation number $\tilde{\rho}(E)=\rho(E)$ and

$$
\left\{\begin{array}{l}
\left|A_{1}(E)\right|<2 \\
\left|\left(\frac{\partial}{\partial E}\right)^{v} A_{1}(E)\right|<\varepsilon_{1}^{-v \sigma}, \quad v=1,2, \\
\left|\left(\frac{\partial}{\partial E}\right)^{v} F_{1}\right|_{r}<\varepsilon_{1}=C(\tau, \sigma) r^{((4 \tau / \sigma)+1)}, \quad v=0,1,2 .
\end{array}\right.
$$

Now A1 follows again from Lemma 2-3. holds.

If $E \leqq-1$, then we are in the resolvent set (if $|q|_{0}<1$ ) and then A1 also

Proof of A2. Suppose that $\tilde{\rho}(\lambda)$ is neither diophantine nor rational, and let $\lambda \in \cap \Lambda_{j}\left(m_{j}\right)$. If $m_{j}=0$ for $j$ large enough, then $X$ has a representation as in A1, so $X$ must be q-p. In this case A2 holds of course.

So suppose there exists an infinite sequence $1 \leqq j_{1}<j_{2}<\cdots$ such that $m_{j_{k}} \neq 0$. Then $\lim A_{j}(\lambda)=0$, by (3.5), so we only need to consider the product $\prod Y_{j}\left(\frac{\omega}{2} t\right)^{j}$.

Let now $t=t_{1}+\cdots+t_{k}, k \geqq 2$ with $\left|t_{l}\right| \leqq 4 \pi N_{j_{l}}^{\tau}$ for all $l$. Choose

$$
t_{k}=\frac{4 \pi}{\left\langle m_{j_{k}}\right\rangle}
$$

and $t_{k-1}$ so that

$$
t_{k-1}+t_{k}=n \frac{4 \pi}{\left\langle m_{j_{k-1}}\right\rangle}, \text { some } n,
$$

etc. Then we get $Z_{j_{1}}(\omega t)=I$ (by construction) and, for all $j_{l}, l \geqq 1$,

$$
\left|Z_{j_{l+1}}(\omega t)-I\right|=\left|Z_{j_{l+1}}\left(\omega T_{l}\right)-I\right|<70 T_{l} N_{j_{l}}^{\tau} \varepsilon_{j_{l}}^{\sigma}
$$

as in (4.1), where

$$
T_{l}=\left\{\begin{array}{ll}
t_{1}+\cdots+t_{l} & l \leqq k-1 \\
t_{1}+\cdots+t_{k} & l \geqq k
\end{array} .\right.
$$

Since

$$
\left|T_{l}\right| \leqq 4 \pi\left(N_{j_{1}}^{\tau}+\cdots+N_{j_{l}}^{\tau}\right) \leqq 4 \pi N_{j_{l}}^{2 \tau},
$$

and since $\left|Y_{j+1}\left(\frac{\omega}{2} t\right)-I\right|<\varepsilon_{j}^{1 / 2}$ for all $j \neq j_{l}$, it follows that

$$
\left|\prod Y_{j}\left(\frac{\omega}{2} t\right)-I\right|<c \varepsilon_{1}^{\sigma / 4}
$$

This gives the first part of A2. 
Let now

$$
\frac{1}{\left|\left\langle m_{j_{k}}\right\rangle\right|} \leqq t<\frac{1}{\mid\left\langle m_{j_{k+1}}\right\rangle}, \quad k \geqq 2
$$

Then

$$
\left|Y_{j+1}\left(\frac{\omega}{2} t\right)-I\right|<c \begin{cases}\varepsilon_{j}^{1 / 2} & j \neq j_{l} \\ N_{j_{l-1}}^{2 \tau} \varepsilon_{j_{l-1}}^{\sigma} & j=j_{l}, \quad l \geqq k+2 \\ \left(1+\left|A_{j}\right|\left|\left\langle m_{j}\right\rangle\right|^{-1}\right) & j=j_{l}, \quad l \leqq k \\ \left(1+\left|t A_{j}\right|\right) & j=j_{k+1} .\end{cases}
$$

(The first estimate is (3.2), the second one is (4.1), and the last two are trivial.) Therefore

$$
\left|\prod_{2}^{k+1} Y_{j_{l+1}}\left(\frac{\omega}{2} t\right)\right| \leqq c^{k} \prod_{2}^{k}\left(\left|\frac{\left\langle m_{j_{l}}\right\rangle}{\left\langle m_{j_{l-1}}\right\rangle}\right|+\left|\frac{A_{j_{l}}}{\left\langle m_{j_{l-1}}\right\rangle}\right|\right) \times\left|\frac{\left\langle m_{j_{1}}\right\rangle}{\left\langle m_{j_{k}}\right\rangle}\right|\left(1+\left|t A_{j_{k+1}}\right|\right),
$$

and since

$$
\left|\frac{\left\langle m_{j_{l}}\right\rangle}{\left\langle m_{j_{l-1}}\right\rangle}\right| \leqq \frac{2 \varepsilon_{j_{l}}^{\sigma}+2\left|A_{j_{l}}\right|}{\left|\left\langle m_{j_{l-1}}\right\rangle\right|} \leqq 70 N_{j_{l-1}}^{2 \tau} \varepsilon_{j_{l-1}}^{\sigma}
$$

by (3.5), the product "converges" to 0 as $k \rightarrow \infty$. Since the last term is bounded by $2\left|\left\langle m_{j_{1}}\right\rangle\right||t|$, this proves $\mathrm{A} 2$.

\section{Coexistence of Quasi-Periodic Solutions-Theorem B1 and C1}

For the proof we shall need two lemmas. For later use we shall give a complex version of the first one even if we in this section only need the real version.

Lemma 4. Suppose $A=A(\lambda) \in s l(2, \mathrm{C})$ is pw. $C^{2}$ on $\Delta$ and let $\pm e(\lambda)$ be the eigenvalues. Then there exists a pw. $C^{2}$ matrix $M=M(\lambda)$ on $\lambda \in \Delta \backslash e^{-1}(0)$ such that $M^{-1} A M=(-i e) J$ and

$$
\begin{aligned}
|M| & \leqq \sqrt{2}, \quad|e(\lambda)||\operatorname{det} M(\lambda)|^{-1} \leqq c|A(\lambda)|, \\
|\partial M| & \leqq c|e|^{-2}|\partial A||A|, \\
\left|\partial^{2} M\right| & \leqq c\left(|e|^{-2}\left|\partial^{2} A\right||A|+|e|^{-4}|\partial A|^{2}|A|^{2}\right) .
\end{aligned}
$$

Moreover, if $A$ and $(-i e)$ are both real, then $M$ can be chosen real.

Proof. Let $A=\left(\begin{array}{cc}a & b \\ c & -a\end{array}\right)$. Suppose that $\left|\begin{array}{l}\frac{a}{c} \\ c\end{array}\right|$ is bounded. Let

$$
V_{1}(\lambda)=\left(\begin{array}{l}
1 \\
0
\end{array}\right), \quad V_{2}(\lambda)=(i e(\lambda))^{-1} A(\lambda) V_{1}(\lambda),
$$

and let $M=\left(V_{-}, V_{+}\right)$, where

$$
V_{-}=\frac{V_{1}}{\sqrt{V_{1}^{2}+V_{2}^{2}}} V_{+}=\frac{V_{2}}{\sqrt{V_{1}^{2}+V_{2}^{2}}}
$$


Since $c \neq 0, M$ is invertible, and since $A^{2}=e^{2} I, M$ conjugates $A$ to $(-i e) J$. If we just observe that $e^{2}=-\operatorname{det} A$, that $\left|V_{1}\right|^{2}+\left|V_{2}\right|^{2} \geqq 1$ and that $|e| \leqq|A|$, then the estimates of $\partial M$ and $\partial^{2} M$ follow readily.

Moreover,

$$
\begin{aligned}
|\operatorname{det} M|^{-1} & =\left|\frac{i e}{c|e|^{2}}\left(|e|^{2}+|a|^{2}+|c|^{2}\right)\right| \\
& \leqq \frac{1}{|e|}\left(\frac{2|a|^{2}+|b c|+|c|^{2}}{|c|}\right) \leqq \text { const. }\left|\frac{A}{e}\right| .
\end{aligned}
$$

The same argument applies if $\left|\frac{b}{c}\right|$ is bounded. On the other hand, if both $\left|\frac{c}{a}\right|$ and $\left|\begin{array}{l}\frac{b}{a} \\ \mid\end{array}\right|$ are small, then the result follows by a perturbation argument.

Let now $A(\lambda) \in \operatorname{sl}(2, \mathbf{R})$ be $C^{2}$ in $\lambda$ in some open set $\Delta \subset \mathbf{R}$. Assume $A(\lambda)=\lambda J+B(\lambda), \lambda \in \Delta$, with

$$
\left|\partial^{v} B(\lambda)\right| \leqq \eta, \quad v=0,1,2 .
$$

Lemma 5. There exists a constant $\eta_{0}$ such that for $\eta<\eta_{0}$ the following hold:

a) $\operatorname{det} A$ has exactly one stationary point $\gamma$. This $\gamma$ is a minimum, $|\gamma|<2 \eta$ and $-6 \eta^{2} \leqq \operatorname{det} A(\gamma) \leqq 0$

b) $\operatorname{det} A$ has exactly two zeros $\lambda_{-} \leqq \lambda_{+}$. Moreover $\left|\lambda_{ \pm}\right|<3 \eta$ and

$$
\frac{1}{3}\left|\lambda_{+}-\lambda_{-}\right| \leqq\left|\lambda_{+}-\gamma\right| \leqq \frac{2}{3}\left|\lambda_{+}-\lambda_{-}\right|
$$

c) the rotation number $\alpha(\lambda)$ of $A(\lambda)$ is monotone and strictly increasing outside $\left[\lambda_{-}, \lambda_{+}\right]$. In particular

$$
\alpha(\lambda)= \begin{cases}\sqrt{\operatorname{det} A(\lambda)} & \lambda_{+} \leqq \lambda \\ 0 & \lambda_{-} \leqq \lambda \leqq \lambda_{+} \\ -\sqrt{\operatorname{det} A(\lambda)} & \lambda \leqq \lambda_{-} ;\end{cases}
$$

d) $\partial \alpha \geqq 1-6 \eta$ outside $\left[\lambda_{-}, \lambda_{+}\right]$;

e) $\frac{1}{4}\left(\lambda_{+}-\lambda_{-}\right) \leqq|A(\lambda)| \leqq 2\left(\lambda_{+}-\lambda_{-}\right), \quad \lambda=\lambda_{-}, \lambda_{+}$.

Proof. Let $f(\lambda)=\operatorname{det} A(\lambda)-\lambda^{2}$. Then it follows that

$$
\left|\partial^{v} f(\lambda)\right| \leqq 2 \eta\left(|\lambda|+2^{v} \eta+v\right)
$$

If $2 \lambda+\partial f(\lambda)=0$, then $\left.\lambda \in \Delta_{1}=\right]-2 \eta, 2 \eta[$. Hence, if there is a stationary point it must lie in $\Delta_{1}$. Since $\partial \operatorname{det} A$ and has different signs at $\pm 2 \eta$, there is a stationary point, and since $\left|\partial^{2} \operatorname{det} A-2\right|<2$ in $\Delta_{1}$, the stationary points is unique, and it is a minimum.

Clearly $-6 \eta^{2} \leqq \operatorname{det} A(\gamma)$, and in order to show that it is $\leqq 0$, it suffices to show that $\operatorname{det} A \leqq 0$ somewhere in $\Delta_{1}$. But this is obvious, since

$$
A(\lambda)=\left(\begin{array}{cc}
a & \lambda+b \\
-\lambda+c & -a
\end{array}\right)
$$

and $-\lambda+c(\lambda)=0$ must have a solution since $|c|<\eta$. This proves a). 
Since the stationary point is unique, there are exactly two zeros, and $\lambda^{2}+f(\lambda)=0$ implies that they lie in $]-3 \eta, 3 \eta[$. In this interval we have $\frac{3}{2} \leqq \partial^{2} \operatorname{det} A \leqq \frac{5}{2}$ which implies that

$$
\frac{3}{4}|\lambda-\gamma|^{2} \leqq|\operatorname{det} A(\lambda)-\operatorname{det} A(\gamma)| \leqq \frac{5}{4}|\lambda-\gamma|^{2},
$$

hence,

$$
\sqrt{\frac{4}{5}|\operatorname{det} A(\gamma)|} \leqq\left|\lambda_{+}-\gamma\right| \leqq \sqrt{\frac{4}{3}|\operatorname{det} A(\gamma)|},
$$

and the same estimate holds for $\left|\lambda_{-}-\gamma\right|$ too. This gives $b$ ).

For $\lambda_{+}<\lambda<1$ we have

$$
(2-7 \eta)(\lambda-\gamma) \leqq \partial \operatorname{det} A(\lambda)=\int_{\gamma}^{\lambda} \partial^{2} \operatorname{det} A d s \leqq(2+7 \eta)(\lambda-\gamma)
$$

and

$$
\operatorname{det} A(\lambda)=\int_{\lambda_{+}}^{\lambda} \partial \operatorname{det} A d s \leqq \frac{1}{2}(2+7 \eta)(\lambda-\gamma)^{2} .
$$

Hence

$$
\partial \alpha(\lambda) \geqq \frac{(2-7 \eta)(\lambda-\gamma)}{\sqrt{2(2+7 \eta)(\lambda-\gamma)^{2}}} \geqq 1-6 \eta .
$$

On the other hand, if $|\lambda| \geqq 1$ then

$$
\partial \alpha(\lambda) \geqq \frac{1+\frac{\partial f}{2 \lambda}}{\sqrt{1+\frac{f}{\lambda^{2}}}} \geqq 1-6 \eta .
$$

This proves d) and now c) is obvious.

Let $A(\lambda)=A_{0}+A_{1}\left(\lambda-\lambda_{+}\right)+\cdots$ with

$$
A_{i}=\left(\begin{array}{cc}
a_{i} & b_{i} \\
c_{i} & -a_{i}
\end{array}\right), \quad i=0,1 .
$$

Notice that $\left|b_{1}-1\right|<\eta,\left|c_{1}+1\right|<\eta$ and all other entries are bounded by $4 \eta$ in norm. Then

and

$$
a_{0}^{2}+b_{0} c_{0}=0
$$

$$
(2-7 \eta)\left(\lambda_{+}-\gamma\right) \leqq\left|-b_{0} c_{1}-b_{1} c_{0}-2 a_{0} a_{1}\right| \leqq(2+7 \eta)\left(\lambda_{+}-\gamma\right) .
$$

The first equality follows from the fact that $\operatorname{det} A=0$ at $\lambda_{+}$, and the second follows from the estimate of $\partial \operatorname{det} A$ given in the proof of d).

If $a_{0}=b_{0}=0$, then

$$
\frac{(2-7 \eta)\left(\lambda_{+}-\gamma\right)}{(1+\eta)} \leqq\left|c_{0}\right| \leqq \frac{(2+7 \eta)\left(\lambda_{+}-\gamma\right)}{(1-\eta)}
$$

which gives the result using b). And the same holds if $a_{0}=c_{0}=0$. 
So assume $a_{0}, b_{0}, c_{0}$ are all $\neq 0$, and let $b_{0}=x a_{0}$ and $c_{0}=-\frac{1}{x} a_{0}$, where we assume, for simplicity, that $x \geqq 1$. Then $\partial \operatorname{det} A\left(\lambda_{+}\right)=a_{0} \delta(x)$, where $\delta(x)=-x c_{1}+$ $\frac{1}{x} b_{1}-2 a_{1} . \delta$ has a unique minimum at $\sqrt{\left|\frac{b_{1}}{c_{1}}\right|}$ for which $\delta \geqq 2-5 \eta$. This gives

$$
\left|a_{0}\right|<\frac{5}{6}\left(\lambda_{+}-\lambda_{-}\right) \text {. }
$$

The same estimate holds for $c_{0}$ since $x \geqq 1$, and for $\frac{b_{0}}{2}$ since $\delta(x) \geqq(1-3 \eta) x$. But $\delta(x) \leqq(2+10 \eta) x$ so we also have

$$
\left|b_{0}\right| \geqq \frac{1}{4}\left(\lambda_{+}-\lambda_{-}\right) .
$$

This proves e).

Corollary 6. If $A_{1}(\lambda)=\lambda J$, then $\partial \alpha_{j}(\lambda) \geqq \prod_{1}^{j-1}\left(1-6 \varepsilon_{k}^{1 / 3}\right)$ when $\alpha_{j}(\lambda) \neq 0$.

Proof. This clearly holds for $j=1$ so we shall assume it for $k \leqq j$ and prove it for $j+1$. $5 d)$.

If $\lambda \in \bigcap_{1}^{j} \Lambda_{l}(0)$, then $\left|\partial^{v}\left(A_{j+1}-A_{1}\right)\right|<2 \varepsilon_{1}^{2 / 3}$ and the result follows from Lemma If $\lambda \in \bigcap_{k+1}^{j} \Lambda_{l}(0) \cap \Lambda_{k}(m), m \neq 0$, then

$$
A_{j+1}-\left(1-\frac{\langle m\rangle}{2 \alpha_{k}}\right) A_{k}=B
$$

with

$$
\left|\partial^{v} B\right|<2 \varepsilon_{k}^{2 / 3}, \quad v=0,1,2 .
$$

Moreover, $\left|\alpha_{k}\right| \geqq \frac{1}{2} N_{k}^{-\tau}$ and $C^{2}$ on some interval $\Delta$ contained in $\Lambda_{k}(m)$, from which we immediately get

$$
\partial^{v} \alpha_{k}<\varepsilon_{k}^{-(3 / 2) v \sigma}, \quad v=1,2,
$$

since $\alpha_{k}= \pm \sqrt{\operatorname{det} A_{k}}$. And, by induction, we also have $\frac{1}{2} \leqq \partial \alpha_{k}$.

By Lemma 4 there exists a $C^{2}$ matrix $M(\lambda)$ on $\Delta$ such that $M^{-1} A_{k} M=\alpha_{k} J$ and satisfying

$$
\left\{\begin{array}{l}
|M| \leqq \sqrt{2} \\
|\operatorname{det} M|^{-1}<\varepsilon_{k}^{-\sigma} \\
\left|\partial^{v} M\right|<\varepsilon_{k}^{-2 v \sigma}, \quad v=1,2
\end{array}\right.
$$

So if $\widetilde{B}=M^{-1} B M$ we get

$$
\left|\partial^{v} \tilde{B}\right|<c \varepsilon_{k}^{2 / 3-(1+3 v) \sigma}
$$

and

$$
\left|\left(\frac{\partial}{\partial \alpha_{k}}\right)^{v} \tilde{B}\right|<c \varepsilon_{k}^{2 / 3-(1+3 v) \sigma}<\varepsilon_{k}^{1 / 3}, \quad v=0,1,2 .
$$


Hence, with $\tilde{A}_{j+1}=M^{-1} A_{j+1} M$ we get

$$
\tilde{A}_{j+1}=\left(\alpha_{k}-\frac{\langle m\rangle}{2}\right) J+\tilde{B}
$$

and we can apply Lemma 5 . Hence

$$
\frac{\partial \alpha_{j+1}}{\partial \alpha_{k}} \geqq 1-6 \varepsilon_{k}^{1 / 3}
$$

if $\alpha_{j+1} \neq 0$. Multiplying by $\partial \alpha_{k}$ and using the estimate, assumed by induction, gives the result.

Proof of Theorem B1 - large energy. Suppose $\tilde{\rho}^{-1}\left(\frac{\langle n\rangle}{2}\right)=\left[\lambda_{-}, \lambda_{+}\right]$, where $\tilde{\rho}$ is defined in Sect. 4. Since we have some freedom in defining the sets $\Lambda_{j}(m)$ we shall choose them in such a way that there exists a decreasing sequence of intervals $\left.\Delta_{j}=\right] a_{j}, b_{j}\left[\supset\left[\lambda_{-}, \lambda_{+}\right]\right.$such that $\alpha_{j}$ is continuous on $\Delta_{j}$,

$$
\rho_{j}(\lambda)=\alpha_{j}(\lambda)+\frac{1}{2} \sum_{k=1}^{j-1}\left\langle m_{k}\right\rangle, \quad \lambda \in \Delta_{j}
$$

and

$$
\left|\rho_{j}(\lambda)-\frac{\langle n\rangle}{2}\right|>\frac{1}{2} \varepsilon_{j}^{\sigma}, \quad \lambda=a_{j}, b_{j} .
$$

Suppose this holds for $k \leqq j$ and let

$$
\left.\Delta_{j+1}=\rho_{j}^{-1}(] \frac{\langle n\rangle}{2}-\frac{\varepsilon_{j}^{\sigma}}{2}, \frac{\langle n\rangle}{2}+\frac{\varepsilon_{j}^{\sigma}}{2}\right) \text {. }
$$

Then $\Delta_{j+1} \supset\left[\lambda_{-}, \lambda_{+}\right]$because $\tilde{\rho}=\frac{\langle n\rangle}{2}$ on $\left[\lambda_{-}, \lambda_{+}\right]$and $\left|\rho_{j}-\tilde{\rho}\right|\left\langle c \varepsilon_{j}^{1 / 4}\right.$ by Lemma 3. Moreover, $\alpha_{j}$ varies precisely $\varepsilon_{j}^{\sigma}$ over $\Delta_{j+1}$, so we can choose the sets $\Lambda_{j}(m)$ in such a way that $\Delta_{j+1} \subset \Lambda_{j}\left(m_{j}\right)$ for some $m_{j}$. Since $\left|\rho_{j+1}-\rho_{j}\right|<c \varepsilon_{j}^{1 / 4}$, also the last condition is fulfilled.

It is now clear that $\bigcap_{j=1}^{\infty} \Delta_{j}=\left[\lambda_{-}, \lambda_{+}\right]$. Moreover, if $\alpha_{j}^{-1}(0) \cap \Delta_{j}=\left[\lambda_{-}^{j}, \lambda_{+}^{j}\right]$, then

$$
\lambda_{ \pm}^{j} \mapsto \lambda_{ \pm}
$$
This follows since $\partial \alpha_{j}=\partial \rho_{j} \geqq \frac{1}{2}$ on $\left[\lambda_{+}^{j}, b_{j}\right]$ and $\left|\rho_{j}-\frac{\langle n\rangle}{2}\right|<c \varepsilon_{j}^{1 / 4}$ on $\left.] \lambda_{+}^{j}, \lambda_{+}\right]$.
The same argument applies to $\lambda_{-}^{j}$.

We now proceed as in the proof of Corollary 6. We assume that $m_{k} \neq 0$. We anti-diagonalize $A_{k}$ by a matrix $M$, and we let $\tilde{A}_{j}=M^{-1} A_{j} M$ for $j>k$. Then

$$
\tilde{A}_{j}=\left(\alpha_{k}-\frac{\left\langle m_{k}\right\rangle}{2}\right) J+\tilde{B}
$$

with

$$
\left|\partial^{v} \tilde{B}\right|<c \varepsilon_{k}^{2 / 3-(1+3 v) \sigma}
$$


and

$$
\left|\left(\frac{\partial}{\partial \alpha_{k}}\right)^{v} \tilde{B}\right|<c \varepsilon_{k}^{2 / 3-(1+3 v) \sigma}<\varepsilon_{k}^{1 / 3}, \quad v=0,1,2 .
$$

So we can apply Lemma 5 to $\tilde{A}_{j}$ as a function of $\alpha_{k}$, and by Lemma 5e) we have

$$
\frac{1}{4}\left|\alpha_{k}\left(\lambda_{+}^{j}\right)-\alpha_{k}\left(\lambda_{-}^{j}\right)\right| \leqq\left|\tilde{A}_{j}\left(\lambda_{ \pm}^{j}\right)\right| \leqq 2\left|\alpha_{k}\left(\lambda_{+}^{j}\right)-\alpha_{k}\left(\lambda_{-}^{j}\right)\right| \text {. }
$$

Since $\frac{1}{2} \leqq \partial \alpha_{k} \leqq N_{k}^{\tau} \varepsilon_{k}^{-\sigma}$ on $] a_{k}, b_{k}[$ it follows that

$$
c_{k}^{-1}\left|\lambda_{+}^{j}-\lambda_{-}^{j}\right| \leqq\left|A_{j}\left(\lambda_{ \pm}^{j}\right)\right| \leqq c_{k}\left|\lambda_{+}^{j}-\lambda_{-}^{j}\right| .
$$

Since $\left|\partial\left(A_{j}-\left(1-\frac{\left\langle m_{k}\right\rangle}{2 \alpha_{k}}\right) A_{k}\right)\right|<2 \varepsilon_{k}^{2 / 3}$ we have a $j$-independent bound for $\partial A_{j}$, and this implies that

$$
c_{k}^{-1}\left|\lambda_{+}-\lambda_{-}\right| \leqq\left|A\left(\lambda_{ \pm}\right)\right| \leqq c_{k}\left|\lambda_{+}-\lambda_{-}\right|
$$

for some constant $c_{k}$. This proves B1 for large energies.

Proof of B1 - small potential. We have

$$
A_{1}(\lambda)=\left(\begin{array}{cc}
0 & 1 \\
-\lambda & 0
\end{array}\right)
$$

with $|\lambda|<1$.

In this case Corollary 6 still holds with $2 \partial \alpha_{j}$ instead of $\partial \alpha_{j}$, and the proof is the same with the only exception that if $\lambda \in \bigcap_{1} \Lambda_{l}(0)$, we cannot apply Lemma $5 \mathrm{~d}$ ).
But then

$$
\left|\partial^{v}\left(A_{j+1}-A_{1}\right)\right|<2 \varepsilon_{1}^{2 / 3}
$$

and

$$
\left|\partial^{v} f\right|<2 \varepsilon_{1}^{2 / 3}(|\lambda|+v+1)
$$

where $f(\lambda)=\operatorname{det} A_{j+1}(\lambda)-\lambda$. Hence,

$$
\partial \alpha_{j+1}=\frac{1+\partial f}{2 \sqrt{\operatorname{det} A_{j+1}}} \geqq \frac{1}{2} \frac{1-6 \varepsilon_{1}^{2 / 3}}{\sqrt{1+6 \varepsilon_{1}^{2 / 3}}} \geqq \frac{1}{2}\left(1-6 \varepsilon_{1}^{1 / 3}\right)
$$

which is what is needed.

The proof of B1 now works in the same way as before. The only additional thing we have to say is that if $]-1, \lambda_{+}\left[\right.$is in the lowest gap then $\left|A\left(\lambda_{+}\right)\right| \geqq 1-2 \varepsilon_{1}^{2 / 3}$. But this is obvious since in that case $\left|A_{j}\left(\lambda_{+}\right)-A_{1}\left(\lambda_{+}\right)\right|<2 \varepsilon_{1}^{2 / 3}$ for all $j$. This proves B1 for small potentials.

Proof of C1. In order to prove the Cantor structure of the spectrum we consider the set $G_{n}$ of all potentials $q$ with $|q|_{r}<\infty$ such that either $\rho^{-1}\left(\frac{\langle n\rangle}{2}, q\right)$ is a gap, or is contained in $]-\infty, E_{0}\left(|q|_{r}\right)+\delta\left[. G_{n}\right.$ is clearly open. But it is also dense. In fact, any collapsed gap in $] E_{0}, \infty$ [ can be opened by an arbitrarily small perturbation, as is described in [2]. 
Hence $G_{n}$ is open and dense, so $\cap G_{n}$ is generic, and if we let $\delta$ go to 0 we obtain that, generically in $] E_{0}, \infty$ [, "all gaps are there." This implies that the spectrum is Cantor because $\rho$ has no other intervals of constancy than the gaps (see [1]).

\section{Unbounded Solutions - Theorem B2}

Let $\lambda \in \cap \Lambda_{j}(m)$ and let $1 \leqq j_{1}<j_{2}, \ldots$ be all indices $j$ for which $m_{j} \neq 0$. Let $t=t_{1}+\cdots$ $+t_{k}, k \geqq 2$, with $\left|t_{l}\right| \leqq 5 \pi N_{j_{l}}^{\tau}$. Choose

$$
t_{k}=\frac{5 \pi}{\left\langle m_{j_{k}}\right\rangle}
$$

and

$$
t_{k-1}+t_{k}=n \frac{4 \pi}{\left\langle m_{j_{k-1}}\right\rangle}, \text { some } n
$$

etc. Then we get, as in the first part of the proof of A2, that $\left|Y_{j+1}\left(\frac{\omega}{2} t\right)-I\right|$ is less than $\varepsilon_{j}^{1 / 2}$ if $j \neq j_{l}$ and less than $c \varepsilon_{j_{l-1}}^{\sigma / 4}$ if $j=j_{l}, l \neq k$. But for $j=j_{k}$ we get

$$
\left|Y_{j+1}\left(\frac{\omega}{2} t\right)-\frac{A_{j_{k}}}{\alpha_{j_{k}}}\right|<c \varepsilon_{j_{k-1}}^{\sigma / 4}\left|\frac{A_{j_{k}}}{\alpha_{j_{k}}}\right| \text {. }
$$

This implies that

$$
\left|\left(\prod_{j} Y_{j}\left(\frac{\omega}{2} t\right)\right)-\frac{A_{j_{k}}}{\alpha_{j_{k}}}\right| \leqq c \varepsilon_{1}^{\sigma / 4}\left(1+\left|\frac{A_{j_{k}}}{\alpha_{j_{k}}}\right|\right) .
$$

Hence, it suffices to construct a $\lambda \in \cap \Lambda_{j}\left(m_{j}\right), m_{j_{k}} \neq 0$, such that the sequence

$$
\left|\frac{A_{j_{k}}(\lambda)}{\alpha_{j_{k}}(\lambda)}\right|
$$

is unbounded, and this we will do now.

Let $\Delta_{1}$ be an open interval. Choose $n_{1}$ such that

$$
\tilde{\rho}^{-1}\left(\frac{\left\langle n_{1}\right\rangle}{2}\right)=\left[\mu_{1}, \lambda_{1}\right] \subset \Delta_{1}, \quad \mu_{1}<\lambda_{1} .
$$

Then $\left|\alpha_{j}\left(\lambda_{1}\right)\right| \rightarrow 0$ and $\left|A_{j}\left(\lambda_{1}\right)\right| \rightarrow\left|A\left(\lambda_{1}\right)\right|=\delta>0$. Choose now $k_{1}$ so large so $8 \varepsilon_{k_{1}}^{1 / 4}<\delta$ and such that

$$
\left\{\begin{array}{l}
\left|\alpha_{k_{1}}\left(\lambda_{1}\right)\right|<\left(\frac{\delta}{8}\right) \\
\left|A_{k_{1}}\left(\lambda_{1}\right)\right| \geqq \delta-\frac{\delta}{8}
\end{array}\right.
$$


Since $\alpha_{k_{1}}$ and $A_{k_{1}}$ are continuous near $\lambda_{1}$ (this can clearly be achieved), it follows that

$$
\left\{\begin{array}{l}
\left|\alpha_{k_{1}}(\lambda)\right|<\left(\frac{\delta}{4}\right) \\
\left|A_{k_{1}}(\lambda)\right| \geqq \delta-\frac{\delta}{4}
\end{array}\right.
$$

for all $\lambda$ in some interval $\left[\lambda_{1}, v_{1}\right] \subset \Delta_{1}$. Let now $j_{1}$ be the largest index $j \geqq k_{1}$ such that $\alpha_{j}$ is continuous on $\left[\lambda_{1}, v_{1}\right]$. (Such a $j$ must exist unless $\lim \alpha_{i}=0$ on $\left[\lambda_{1}, v_{1}\right]$. But this would imply that $\rho$ is constant on $\left[\lambda_{1}, v_{1}\right]$ which is impossible since $\lambda_{1}$ is the right endpoint of a gap.) Then

$$
\left|\alpha_{j_{1}}-\alpha_{k_{1}}\right|<c \varepsilon_{k_{1}}^{1 / 4}, \quad\left|A_{j_{1}}-A_{k_{1}}\right|<2 \varepsilon_{k_{1}}^{1 / 2}
$$

on $\left[\lambda_{1}, v_{1}\right]$. Hence,

$$
\begin{aligned}
& \left|\alpha_{j_{1}}(\lambda)\right|<\left(\frac{\delta}{2}\right) \\
& \left|A_{j_{1}}(\lambda)\right| \geqq \delta-\frac{\delta}{2}
\end{aligned}
$$

on $\left[\lambda_{1}, v_{1}\right]$, and, by the choice of $j_{1}$, there exists an $m_{j_{1}}, 0<\left|m_{j_{1}}\right| \leqq N_{j_{1}}$ with

$$
\left|2 \alpha_{j_{1}}(\lambda)-\left\langle m_{j_{1}}\right\rangle\right|<2 \varepsilon_{j_{1}}^{\sigma}
$$

for some $\lambda$ in this interval.

But this implies that $\left.\Lambda_{j_{1}}\left(m_{j_{1}}\right) \cap\right] \lambda_{1}, v_{1}$ [ contains a non-void open interval $\Delta_{2}$. Now we can repeat this construction with a gap $\left[\mu_{2}, \lambda_{2}\right]$ in $\Delta_{2}$, and find a $j_{2}$ such that

$$
\left\{\begin{array}{l}
\left|\alpha_{j_{2}}(\lambda)\right|<\frac{\delta}{2^{2}} \\
\left|A_{j_{2}}(\lambda)\right| \geqq \delta-\frac{\delta}{2^{2}}
\end{array}\right.
$$

on some interval $\left[\lambda_{2}, v_{2}\right]$, and such that there exists an $m_{j_{2}}, 0<\left|m_{j_{2}}\right| \leqq N_{j_{2}}$ with

$$
\left|2 \alpha_{j_{2}}(\lambda)-\left\langle m_{j_{2}}\right\rangle\right|<\varepsilon_{j_{2}}^{\sigma}
$$

for some $\lambda$ in this interval.

In this way we construct a decreasing sequence of intervals

$$
\Delta_{1} \supset \Delta_{2} \supset \cdots
$$

which contains a point $\lambda \in \cap \Lambda_{j_{l}}\left(m_{j_{l}}\right)$ such that

$$
\left|\frac{A_{j_{l}}(\lambda)}{\alpha_{j_{l}}(\lambda)}\right| \geqq \frac{\delta-\frac{\delta}{2^{l}}}{\frac{\delta}{2^{l}}}=2^{l}-1
$$

This proves $\mathrm{B} 2$. 


\section{Solutions for Complex Energies $E+i g$}

We shall only consider the case with $E$ large, and then we indicate what modifications are required in order to treat also small $E$ when the potential is small.

The equation $(* *)$ gets transformed to

$$
X_{1}^{\prime}(t)=\left(A_{1}(\sqrt{E+i g})+F_{1}(\omega t, \sqrt{E+i g})\right) X_{1}(t),
$$

where $A_{1}(\sqrt{E+i g})=\sqrt{E+i g} J$ and

$$
F_{1}(x, \sqrt{E+i g})=\frac{q(x)}{2 \sqrt{E+i g}}\left(\begin{array}{ll}
-1 & -1 \\
+1 & +1
\end{array}\right)
$$

through the change of variables $X_{1}=Y_{1}^{-1} X$, where

$$
Y_{1}=\left(\begin{array}{cc}
1 & 1 \\
-\sqrt{E+i g} & \sqrt{E+i g}
\end{array}\right) .
$$

We have

$$
\begin{cases}\left|A_{1}-\sqrt{E+i g} J\right|<2 & v=1,2 \\ \left|\partial A_{1}\right|<\varepsilon_{1}^{-v \sigma} & v=0,1,2 \\ \left|\partial^{v} F_{1}\right|_{r_{1}}<\frac{|q|_{r_{1}}}{\sqrt{E}}<\varepsilon_{1} & \end{cases}
$$

if $E$ is large enough, where $\partial=\frac{\partial}{\partial \lambda}=\sqrt{E} \frac{\partial}{\partial g}$.

Let $N_{j}$ and $\varepsilon_{j}$ be as in Lemma 2. Let $\sqrt{E} \in \bigcap_{j \geqq 1} \Lambda_{j}\left(m_{j}\right), E>E_{0}$, such that $\alpha(\sqrt{E}) \neq 0$. This implies that all $m_{j}$, except finitely many, are 0 , so we let $1 \leqq j_{1}<\cdots<j_{n}$ be all the indices for which $m_{j} \neq 0$ - if no such indices exist we let $n=0$. We shall let $k$ be the smallest integer $\geqq j_{n}$ such that

$$
\varepsilon_{j}^{\sigma^{2}} N_{j}^{6 \tau} \leqq 1, \quad j \geqq k
$$

Lemma 7. There exists a constant $C=C(\tau, \sigma)$ such that if $\varepsilon_{1}<C r_{1}^{((4 \tau / \sigma)+1)}$ then, for all $j \geqq 1$, there exist $F_{j+1}, Y_{j+1} \in \mathscr{B}, \operatorname{tr}\left\langle F_{j+1}\right\rangle=0$, and $A_{j+1} \in \operatorname{sl}(2, \mathbf{C})$ such that

$$
\left\langle Y_{j+1}^{\prime}\left(\frac{x}{2}\right), \frac{\omega}{2}\right\rangle=\left(A_{j}+F_{j}(x)\right) Y_{j+1}\left(\frac{x}{2}\right)-Y_{j+1}\left(\frac{x}{2}\right)\left(A_{j+1}+F_{j+1}(x)\right) \text {. }
$$

Moreover, $F_{j+1}, Y_{j+1}, A_{j+1}$ are $C^{2}$ for $|\lambda|<\varepsilon_{k+1}^{\sigma}$, real when $\lambda=0$, and their first derivatives with respect to $\lambda$ are purely imaginary at $\lambda=0$.

Let $e_{j}(\sqrt{E+i g})$ be a continuous choice of the eigenvalue of $A_{j}$ which we determine by the condition that $\operatorname{Im} e_{j}(\sqrt{E})=\alpha_{j}(\sqrt{E})$ - this determination is unique if $\alpha_{j} \neq 0$.

Let $r_{j}-r_{j+1}=\frac{1}{2} r_{j}$ if $m_{j} \neq 0$ and $r_{j}-r_{j+1}=2^{-j} \frac{r_{1}}{2}$ if $m_{j}=0$. Then we have the following estimates.

$$
\begin{aligned}
\left|\partial^{v}\left(Y_{j+1}-e^{\left.i\left(\left\langle m_{j}, .\right\rangle\right) / 2 e_{j}\right) A_{j}}\right)\right|_{r_{j+1}}<\varepsilon_{j}^{1 / 2}, & v=0,1,2 ; \\
\left|\partial^{v} F_{j+1}\right|_{r_{j+1}}<\varepsilon_{j+1}, & v=0,1,2 ;
\end{aligned}
$$




$$
\begin{aligned}
\left|\partial^{v}\left(A_{j+1}-\left(1-i \frac{\left\langle m_{j}\right\rangle}{2 e_{j}}\right) A_{j}\right)\right|<\varepsilon_{j}^{2 / 3}, \quad v=0,1,2 ; \\
\begin{cases}\left|\partial^{v} A_{j+1}\right|<\varepsilon_{j+1}^{-v \sigma}, & v=1,2 \\
\left|A_{j+1}\right|<32\left|e_{j+1}\right| N_{j+1}^{\tau} & \text { if }\left|e_{j+1}\right| \geqq \frac{1}{4} N_{j+1}^{-\tau} .\end{cases}
\end{aligned}
$$

This lemma has one different aspect from Lemma 2. In Lemma 2 the whole construction depends sensitively on the parameter $\lambda=\sqrt{E}$. Changing $\lambda$ implies that the sequence $\cap \Lambda_{j}\left(m_{j}\right)$ and, hence, all the estimates change. In Lemma 7 the sequence $\cap \Lambda_{j}\left(m_{j}\right)$ does not change when we vary $\lambda=\frac{g}{\sqrt{E}}$ over a small interval of length $\varepsilon_{k+1}^{\sigma}$. In order to see this, we only have to check certain properties of $e_{j}$.

So suppose we have constructed $Y_{j}, F_{j}, A_{j}$. We shall verify that

$$
\left|\operatorname{Re} e_{j}(\sqrt{E+i g})\right| \geqq \frac{1}{4}\left|\frac{g}{\sqrt{E}}\right|,
$$

and that, for $|\lambda|<\varepsilon_{k+1}^{\sigma}$,

$$
\begin{cases}e_{j}(\sqrt{E+i g})-i \frac{\langle m\rangle}{2}\left|\geqq \frac{1}{4} \varepsilon_{j}^{\sigma}, \quad 0<\right| m \mid \leqq N_{j}, & \text { if } \quad m_{j}=0 \\ e_{j}(\sqrt{E+i g})-i \frac{\left\langle m_{j}\right\rangle}{2} \mid \leqq 2 \varepsilon_{j}^{\sigma}, & \text { if } \quad m_{j} \neq 0 .\end{cases}
$$

Then we can proceed as in the proof of Lemma 2 in order to construct the $(j+1)^{\text {th }}$ step. To prove (7.5), we observe that $e_{1}(\sqrt{E+i g})=i \sqrt{E+i g}=-\frac{g}{2 \sqrt{E}}+i \sqrt{E}+O^{2}(\lambda)$,
so $(7.5)_{1}$ holds, and we then proceed by induction.

Let $l$ be the largest integer $<j$ for which $m_{l} \neq 0$. Clearly $l \leqq k$ and by (7.3)

$$
A_{j}=\left(1-i \frac{\left\langle m_{l}\right\rangle}{2 e_{l}}\right) A_{l}+B
$$

with $\left|\partial^{v} B\right|<2 \varepsilon_{l}^{2 / 3}, v=0,1$. Let $M$ be a matrix such that

$$
\begin{array}{r}
M^{-1} A_{l} M=\left(-i e_{l}\right) J, \quad|M| \leqq \sqrt{2} \\
|\operatorname{det} M|^{-1} \leqq c N_{l}^{\tau}, \quad|\partial M| \leqq c N_{l}^{2 \tau} \varepsilon_{l}^{-\sigma},
\end{array}
$$

and $M$ being real when $g=0$ - such a matrix exists by Lemma 4 . Then

$$
M A_{j} M^{-1}=-i\left(e_{l}-i \frac{\left\langle m_{l}\right\rangle}{2}\right) J+\tilde{B}
$$

with $\left|\partial^{v} \tilde{B}\right|<2 \varepsilon_{l}^{2 / 3-2 \sigma}, v=0,1$.

If now $\widetilde{B}(0)=0$, then $\widetilde{B}$ is small compared with $\left(e_{l}-i \frac{\left\langle m_{l}\right\rangle}{2}\right)$ by $(7.5)_{l}$, and then it is an easy perturbation result to show that $\left|e_{j}-\left(e_{l}-i \frac{\left\langle m_{l}\right\rangle}{2}\right)\right|<\varepsilon_{l}^{1 / 2}$. We 
can therefore assume that $\tilde{B}=\widetilde{B}(0)$ is independent of $g$, and then the result follows by an explicit computation. In fact, if $z=e_{l}-i \frac{\left\langle m_{l}\right\rangle}{2}$ and $\widetilde{B}=\left(\begin{array}{cc}a & b \\ c & -a\end{array}\right)$, then

$$
\operatorname{det} A_{l}=\left(i z+\frac{c-b}{2}\right)^{2}-\left(a^{2}+\left(\frac{b+c}{2}\right)^{2}\right) \text {. }
$$

But if $(u+i v)^{2}=(x+i y)^{2}-d^{2}$ then clearly $v^{2} \geqq y^{2}$, and this shows that $\operatorname{Re} e_{j} \geqq$ $\operatorname{Re} e_{l} \geqq 0$ or $\operatorname{Re} e_{j} \leqq \operatorname{Re} e_{l} \leqq 0$.

Finally, if there is no such integer $l$ as assumed above, then $A_{j}(\sqrt{E}) \sim \sqrt{E} J$ and $\alpha_{j}(\sqrt{E}) \sim \sqrt{E}$ and $(7.5)_{j}$ is an easy perturbation result.

To prove (7.6) $)_{j}$ we observe that if $|\lambda|>\varepsilon_{j}^{\sigma}$, then $j>k$ and $m_{j}=0$, and we are done by $(7.5)_{j}$. So we can assume that $|\lambda|<\varepsilon_{j}^{\sigma}$.

Now

$$
A_{j}(\sqrt{E+i g})=A_{j}(\sqrt{E})+i B_{1} \lambda+B_{2}(\lambda) \lambda^{2}
$$

with $B_{1}$ real. Let $l$ be the largest integer $<j$ such that $m_{l} \neq 0$. Then, by (7.3),

$$
\left|B_{v}\right| \leqq c\left(N_{l}^{2 \tau} \varepsilon_{l}^{-\sigma}\right)^{v}, \quad v=1,2,
$$

and hence

$$
\operatorname{det} A_{j}(\sqrt{E+i g})=\alpha_{j}^{2}(\sqrt{E})+i a \lambda+b(\lambda) \lambda^{2}=\alpha_{j}^{2}(\sqrt{E})\left[1+\frac{i a \lambda}{\alpha_{j}^{2}(\sqrt{E})}+\frac{b(\lambda) \lambda^{2}}{\alpha_{j}^{2}(\sqrt{E})}\right]
$$

with $a$ real. Since $\left|A_{j}(\sqrt{E})\right| \leqq c \varepsilon_{l}^{\sigma} N_{l}^{\tau}$, it follows that

$$
|a|<c N_{l}^{3 \tau}, \quad|b|<c N_{l}^{4 \tau} \varepsilon_{l}^{-2 \sigma} .
$$

If now $\alpha_{j}$ is small, $\left|\alpha_{j}\right|<\frac{1}{4} N_{j}^{-\tau}$ say, then $m_{j}=0$ and

$$
\left|\operatorname{det} A_{j}(\sqrt{E+i g})\right|<\frac{1}{8} N_{j}^{-2 \tau},
$$

which implies (7.6) ${ }_{j}$. So let's suppose that $\frac{1}{4} N_{j}^{-\tau} \leqq\left|\alpha_{j}\right| \leqq \varepsilon_{l}^{\sigma} N_{l}^{\tau}$.

If now $j \leqq k$, then

$$
\left|\frac{a \lambda}{\alpha_{j}}\right| \leqq c \varepsilon_{k+1}^{\sigma} N_{j}^{\tau} N_{l}^{3 \tau}<\frac{1}{2} \varepsilon_{j}^{\sigma}
$$

and

$$
\left|\frac{b \lambda^{2}}{\alpha_{j}}\right| \leqq c \varepsilon_{k+1}^{2 \sigma} N_{j}^{\tau} N_{l}^{4 \tau} \varepsilon_{l}^{-2 \sigma} \leqq c \varepsilon_{k+1}^{2 \sigma} N_{j}^{3 \tau} N_{l}^{6 \tau} \leqq \frac{1}{2} \varepsilon_{j}^{\sigma},
$$

since $\varepsilon_{k}^{\sigma^{2}} N_{k}^{6 \tau} \leqq 1$.

On the other hand, if $j>k$, then $\left|\frac{b \lambda^{2}}{\alpha_{j}}\right|$ is still less than $\varepsilon_{j}^{\sigma}$. Moreover,

$$
\left|\frac{a \lambda}{\alpha_{j}}\right|^{2} \leqq \varepsilon_{j}^{\sigma}
$$


which implies that

$$
\left|e_{j}(\sqrt{E+i g})-i \alpha_{j}(\sqrt{E})-\frac{a \lambda}{\alpha_{j}(\sqrt{E})}\right|<\varepsilon_{j}^{\sigma} .
$$

This proves the result since $m_{j}=0$ and $a$ is real.

Finally, if there is no such integer $l$ as assumed above, then $A_{j}(\sqrt{E}) \sim \sqrt{E} J$ and $\alpha_{j}(\sqrt{E}) \sim \sqrt{E}$ and $(7.6)_{j}$ is an easy perturbation result. Then

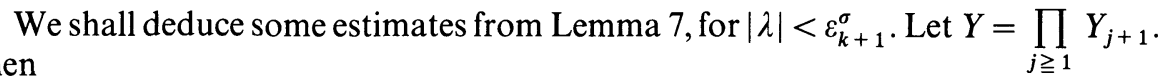

$$
\begin{gathered}
|Y|_{0}<c \begin{cases}N_{j_{n}}^{\tau} & n \neq 0 \\
1 & n=0\end{cases} \\
|\partial Y|_{0}<c \begin{cases}\varepsilon_{j_{n}}^{-2 \sigma} & n \neq 0 \\
\varepsilon_{1}^{1 / 2} & n=0 .\end{cases}
\end{gathered}
$$

In order to see this, observe that $\left|Y_{j+1}\right|_{0} \leqq c\left|\frac{A_{j}}{e_{j}}\right|$ if $j=j_{l}$. Hence

$$
\begin{aligned}
|Y|_{0} & \leqq c^{n}\left|A_{j_{1}}\right| \cdots\left|A_{j_{n}}\right|\left(\left|e_{j_{1}}\right| \cdots\left|e_{j_{n}}\right|\right)^{-1} \\
& \leqq c^{n}\left|\frac{A_{j_{1}}}{e_{j_{1}}}\right|\left(N_{j_{1}}^{\tau} \varepsilon_{j_{1}}^{\sigma} \cdots N_{j_{n-1}}^{\tau} \varepsilon_{j_{n-1}}^{\sigma}\right) N_{j_{2}}^{\tau} \cdots N_{j_{n}}^{\tau},
\end{aligned}
$$

which follows just as (3.5) follows from Lemma 2. Now $\left|\frac{A_{j_{1}}}{e_{j_{1}}}\right|<N_{j_{1}}^{\tau}$ by (7.4) which gives the result. Also $\left|\partial Y_{j+1}\right|_{0} \leqq N_{j}^{2 \tau} \varepsilon_{j}^{-\sigma}$ for $j=j_{l}$ which implies

$$
\begin{aligned}
|\partial Y|_{0} & <c\left(N_{j_{n}}^{\tau} \varepsilon_{1}^{1 / 2}+N_{j_{n}}^{\tau}\left(N_{j_{1}}^{2 \tau} \varepsilon_{j_{1}}^{-\sigma}+\cdots+N_{j_{n}}^{2 \tau} \varepsilon_{j_{n}}^{-\sigma}\right)\right) \\
& <c N_{j_{n}}^{3 \tau} \varepsilon_{j_{n}}^{-\sigma}<\varepsilon_{j_{n}}^{-2 \sigma} .
\end{aligned}
$$

Of course, if $n=0$, then these estimates are obvious.

Let now $A=\lim A_{j}$. Then it follows from Lemma 7 that

$$
\begin{cases}|A(\sqrt{E+i g})|<c \varepsilon_{j_{n}}^{\sigma} N_{j_{n}}^{\tau} & n \neq 0 \\ |A(\sqrt{E+i g})-\sqrt{E+i g} J|<2 \varepsilon_{1}^{1 / 2} & n=0\end{cases}
$$

and

$$
|\partial A(\sqrt{E+i g})| \leqq c \begin{cases}\varepsilon_{j_{n}}^{-\sigma} N_{j_{n}}^{2 \tau} & n \neq 0 \\ 1 & n=0\end{cases}
$$

For $n \geqq 1$ we get from (7.10) that

and from (7.9) that

$$
|A(\sqrt{E+i g})-A(\sqrt{E})|<c \varepsilon_{j_{n}}^{-\sigma} N_{j_{n}}^{2 \tau}|\lambda|
$$

$$
|\operatorname{det} A(\sqrt{E+i g})-\operatorname{det} A(\sqrt{E})|<c \varepsilon_{j_{n}}^{\sigma} N_{j_{n}}^{\tau} \varepsilon_{j_{n}}^{-\sigma} N_{j_{n}}^{2 \tau}|\lambda|<\varepsilon_{j_{n}}^{-\sigma}|\lambda| .
$$

For $n=0$ we get in the same way an estimate by $c \sqrt{E}|\lambda|$. Hence,

$$
|e(\sqrt{E+i g})-e(\sqrt{E})| \leqq c \begin{cases}\sqrt{\varepsilon_{j_{n}}^{-\sigma}|\lambda|} & n \neq 0 \\ \sqrt{|\lambda|} & n=0\end{cases}
$$


For $|\lambda|<\varepsilon_{k+1}^{\sigma} \min \left\{1,|\alpha(\sqrt{E})|^{2}\right\}$ it follows immediately from (7.9) and (7.11) that

$$
\left|\frac{A}{e}(\sqrt{E+i g})\right|<c \begin{cases}\frac{\varepsilon_{j_{n}}^{\sigma} N_{j_{n}}^{\tau}}{|\alpha(\sqrt{E})|} & n \neq 0 \\ 1 & n=0 .\end{cases}
$$

Let now $M$ be a matrix such that

$$
\begin{gathered}
M^{-1} A M=e\left(\begin{array}{cc}
1 & 0 \\
0 & -1
\end{array}\right), \quad|M| \leqq \sqrt{2} \\
|\operatorname{det} M|^{-1} \leqq c\left|\frac{A}{e}\right|, \quad|\partial M| \leqq c\left|\frac{A}{e}\right|\left|\frac{\partial A}{e}\right| .
\end{gathered}
$$
The existence of $M$ follows from Lemma 4 , since we can conjugate $J$ to $i\left(\begin{array}{cc}1 & 0 \\ 0 & -1\end{array}\right)$
by a fixed non-singular matrix.

For $|\lambda|<\varepsilon_{k+1}^{\sigma} \min \left\{1,|\alpha(\sqrt{E})|^{2}\right\}$ we have

$$
\begin{array}{r}
|\operatorname{det} M|^{-1} \leqq c \begin{cases}\frac{\varepsilon_{j_{n}}^{\sigma} N_{j_{n}}^{\tau}}{|\alpha(\sqrt{E})|} & n \neq 0 \\
1 & n=0\end{cases} \\
\left|\partial(\operatorname{det} M)^{-1}\right| \leqq c \begin{cases}\frac{\varepsilon_{j_{n}}^{2 \sigma} N_{j_{n}}^{5 \tau}}{|\alpha(\sqrt{E})|^{4}} & n \neq 0 \\
\frac{1}{\sqrt{E}} & n=0 .\end{cases}
\end{array}
$$

This follows immediately from (7.10-12).

Let now $X=Y e^{A t} M$. For $|\lambda|<\varepsilon_{k+1}^{\sigma} \min \left\{1,|\alpha(\sqrt{E})|^{2}\right\}$ we have

$$
\begin{aligned}
& |X(t)| \leqq c_{t} \begin{cases}N_{j_{n}}^{\tau} & n \neq 0 \\
1 & n=0\end{cases} \\
& |\partial X(t)| \leqq c_{t} \begin{cases}\varepsilon_{j_{n}}^{-\sigma}\left(\varepsilon_{j_{n}}^{-\sigma}+|\alpha(\sqrt{E})|^{-2}\right) & n \neq 0 \\
1 & n=0,\end{cases}
\end{aligned}
$$

where the constant $c_{t}$ depends on $t$.

This follows if we can estimate $e^{A t}$. If $n \neq 0$ then $|A|<1$ and $\left|\partial\left(e^{A t}\right)\right| \leqq e^{|A t|}|t||\partial A|$, and we are done. If $n=0$, then

$$
e^{A t}=M^{-1}\left[\exp \left(\begin{array}{cc}
\text { et } & 0 \\
0 & -\mathrm{et}
\end{array}\right)\right]_{M}
$$

and, since $|e-i \sqrt{E}|<1$, we get $\left|e^{A t}\right| \leqq e^{2|t|}$. And $\left|\partial e^{A t}\right| \leqq c(|t|+1) e^{|t|}$.

Remark. In the case of small potentials, the equation $(* *)$ is

$$
X_{1}^{\prime}(t)=\left(A_{1}(E+i g)+F_{1}(\omega t, E+i g)\right) X_{1}(t),
$$


where

$$
A_{1}=\left(\begin{array}{cc}
0 & 1 \\
-(E+i g) & 0
\end{array}\right), \quad F_{1}(x, E+i g)=\left(\begin{array}{cc}
0 & 0 \\
q(x) & 0
\end{array}\right) .
$$

For $|E|<1$ we have

$$
\begin{cases}\left|A_{1}\right|<2 & \\ \left|\partial^{v} A_{1}\right|<\varepsilon_{1}^{-v \sigma}, & v=1,2, \\ \left|\partial^{v} F_{1}\right|_{r_{1}}<|q|_{r_{1}}<\varepsilon_{1}, & v=0,1,2\end{cases}
$$

if $|q|_{r_{1}}$ is small enough. Here $\partial=\frac{\partial}{\partial \lambda}$, where $\lambda=g$.

Now everything goes true in essentially the same way. Of course, the argument in no longer $\sqrt{E+i g}$, but simply $E+i g$. Equation (7.6) remains true, while (7.5) takes the form

$$
\left|\operatorname{Re} e_{j}(E+i g)\right| \geqq \frac{1}{4}|g| \text {. }
$$

The modification of the proof is very small and concerns only the case when $n=0-$ all other cases being the same.

The estimates (7.7-8) remains true, as well as (7.9), with an obvious modification for the case $n=0$. (7.10-11) remains true. For (7.12) the only difference is that when $n=0$, the estimate is not 1 but $\frac{1}{|\alpha(E)|}$. Also the estimates $(7.13-14)$ are the same when $n \neq 0$ while they are different if $n=0$. If $n=0$ then $|\operatorname{det} M|^{-1} \leqq c|\alpha(E)|^{-1}$, $\left|\partial(\operatorname{det} M)^{-1}\right| \leqq c|\alpha(E)|^{-3},|X(t)| \leqq c_{t}$, and $|\partial X(t)| \leqq c_{t}\left(1+|\alpha(E)|^{-2}\right)$.

\section{Absolutely Continuous Spectrum}

Let $\phi \in C_{c}^{\infty}(\mathbf{R})$ be real, and define

$$
\mu(E)=\mu_{\phi}(E)=\lim _{\delta \searrow 0} \lim _{g \searrow 0} \frac{1}{\pi} \int_{-\infty}^{E+\delta} \operatorname{Im}\left\langle\phi,(\overline{\mathscr{L}}-(s+i g))^{-1} \phi\right\rangle d s .
$$

It's a general fact for all self adjoint operators $\overline{\mathscr{L}}$ that $\mu$ is right continuous and increasing, and that it has a discontinuity at $E_{1}$ if and only if $E_{1}$ is an eigenvalue of $\overline{\mathscr{L}}$. Moreover.

$$
\operatorname{Im}\left\langle\phi,(\overline{\mathscr{L}}-(E+i g))^{-1} \phi\right\rangle=\int_{-\infty}^{+\infty} \frac{g}{(E-s)^{2}+g^{2}} d \mu(s), \quad g>0
$$

and

$$
\lim _{g \searrow 0} \operatorname{Im}\left\langle\phi,(\overline{\mathscr{L}}-(E+i g))^{-1} \phi\right\rangle=r(E)=\frac{d \mu}{d E}(E),
$$

the pointwise derivative of $\mu$, for a.e. $E \in \mathbf{R}$. (See $[13,14]$.) Moreover, $r$ is locally integrable by the Fatou lemma.

Since $\overline{\mathscr{L}}$ is a Sturm-Liouville operator with $C^{\infty}$ coefficients, any eigenfunction of $\overline{\mathscr{L}}$ is $C^{\infty}$ and, hence, an eigenfunction of $\mathscr{L}$ (see [12]). By Theorem A no such eigenfunctions exist for $E>E_{0}$, so $\mu(E)$ must be continuous on $] E_{0}, \infty$ [. (In particular, we can let $\delta$ be 0 in the formulas above.) In this section we shall show that $\mu$ is 
absolutely continuous on $] E_{0}, \infty[$. We shall only do the details for large $E$ - for a small potential the proof is essentially the same.

The resolvent of $\overline{\mathscr{L}}$ has a representation

$$
\begin{aligned}
(\overline{\mathscr{L}}-(E+i g))^{-1} \phi(t) & =y_{+}(t) \int_{-\infty}^{t} \frac{y_{-}(s) \phi(s)}{W\left(y_{+}, y_{-}\right)} d s+y_{-}(t) \int_{t}^{+\infty} \frac{y_{+}(s) \phi(s)}{W\left(y_{+}, y_{-}\right)} d s \\
W\left(y_{+}, y_{-}\right) & =\operatorname{det}\left(\begin{array}{ll}
y_{+} & y_{-} \\
y_{+}^{\prime} & y_{-}^{\prime}
\end{array}\right)(0)
\end{aligned}
$$

for any pair of Weyl solutions $y_{ \pm}(t)$, i.e. solutions such that

$$
\int_{0}^{\infty}\left|y_{+}(t)\right|^{2} d t<+\infty, \quad \int_{-\infty}^{0}\left|y_{-}(t)\right|^{2} d t<+\infty .
$$

We shall express the resolvent in terms of the Floquet solutions constructed in Sect. 7.

Let $E$ be such that $\sqrt{E} \in \bigcap_{j \geqq 1} \Lambda_{j}\left(m_{j}\right)$ with $\alpha(\sqrt{E}) \neq 0$ - this holds if, for example, $\rho(E)$ is diophantine. This implies that $m_{j}=0$ for all $j>j_{n}$. Let $Y=\prod_{j \geqq 1} Y_{j+1}$ and $A=\lim A_{j}$, with $Y_{j}, A_{j}$ given by Lemma 7 , and let $M$ be a matrix as in (7.13). If we take

$$
\left(\begin{array}{ll}
y_{+} & y_{-} \\
y_{+}^{\prime} & y_{-}^{\prime}
\end{array}\right)(t)=Y_{1} X(t)=Y_{1} Y e^{A t} M
$$

where

$$
Y_{1}=\left(\begin{array}{cc}
1 & 1 \\
-\sqrt{E+i g} & +\sqrt{E+i g}
\end{array}\right),
$$

then $y_{ \pm}$is a pair of Weyl solutions if $g \neq 0$, by (7.5). Using these solutions we can define the limit $(\overline{\mathscr{L}}-E)^{-1} \phi(t)$. Then we get the following results by an application of the estimates (7.13-14).

Choose $k$ so large so $m_{j}=0$ and $\varepsilon_{j}^{\sigma^{2}} N_{j}^{6 \sigma} \leqq 1$ for $j>k$. For

$$
|g|<\varepsilon_{k+1}^{\sigma} \min \left\{1,|\alpha(\sqrt{E})|^{2}\right\} \text { and } t \in \operatorname{supp} \phi
$$

we have

$$
\left|(\overline{\mathscr{L}}-(E+i g))^{-1} \phi(t)\right| \leqq c_{\phi} \begin{cases}\sqrt{E} \varepsilon_{j_{n}}^{\sigma} N_{j_{n}}^{3 \tau} \frac{1}{|\alpha(\sqrt{E})|} & n \neq 0 \\ \sqrt{E} & n=0\end{cases}
$$

and

$$
\left|\left((\overline{\mathscr{L}}-(E+i g))^{-1}-(\overline{\mathscr{L}}-E)^{-1}\right) \phi(t)\right| \leqq c_{\phi} \begin{cases}E \varepsilon_{j_{n}}^{-\sigma} N_{j_{n}}^{2 \tau}\left(1+|\alpha(\sqrt{E})|^{-4}\right)|g| & n \neq 0 \\ E|g| & n=0\end{cases}
$$

where $c_{\phi}$ is a constant that depends on $\sup |\phi(t)|$ and on $\operatorname{supp}(\phi)$. 
From (8.1-2) it follows that $d \mu$ is absolutely continuous on the set $D=\bigcup_{l \geqq 1} D_{l}$,

$$
D_{l}=\left\{E>E_{0}:|\alpha(E)|>\frac{1}{l}\right\} .
$$

This is so because on $D_{l}$ the integer $j_{n}$ in the formulas (8.1-2) is uniformly bounded, so we have uniform convergence

$$
\operatorname{Im}\left\langle\phi,(\overline{\mathscr{L}}-(E+i g))^{-1} \phi\right\rangle \rightarrow r(E), \quad g \rightarrow 0,
$$

and the limit function is uniformly bounded. (In order to see that this implies the absolute continuity on $D_{l}$ one can apply the argument of [3], which we shall use below in a more delicate situation.)

So the problem is the set of points $E$ for which $\alpha(\sqrt{E})=0$. Of course this set includes the gaps, which should be excluded, as well as the endpoints of the gaps which we can exclude because there are only countably many such points. So we let

$$
S=\left\{E>E_{0}: \alpha(\sqrt{E})=0\right\} \cap \rho^{-1}(\mathbf{R} \backslash \mathbf{Q}) .
$$

We shall prove

Lemma 8. Let $E \in S$. Then, for any $\delta<\delta_{0}(E)$, there exists an open interval $I=I(E, \delta)$ containing $E$ such that $|I|<\delta$ and

$$
d \mu(I) \leqq 4 \int_{I} r(s) d s+2|I|
$$

where $|I|$ denotes the Lebesgue measure.

This implies that for any interval $\Delta$

$$
d \mu(S \cap \Delta) \leqq 2\left(4 \int_{\Delta} r(\mu) d \mu+2|\Delta|\right)
$$

- an excercise - and hence the absolute continuity of $d \mu$ since $r$ is locally integrable. So we are left with the

Proof of Lemma 8. Let $E_{1} \in S$. This implies that $\sqrt{E_{1}} \in \cap \Lambda_{l}\left(m_{l}\right)$ with $m_{l} \neq 0$ for infinitely many $l$ :s. Let $k$ and $j$ be two consecutive indices such that $m_{k}$ and $m_{j}$ both are non-zero, and let $k$ be so large so $\varepsilon_{l}^{\sigma^{2}} N_{l}^{6 \tau} \leqq 1, l \geqq k$.

Let $\Delta$ be a symmetric interval around $\sqrt{E_{1}}$ of diameter $|\Delta|=c_{\phi} E_{1}^{-3 / 2} \varepsilon_{k}^{2 \sigma} N_{j}^{-4 \tau}$. It follows from (3.7) that if $k$ is large enough - depending on $E_{1}$ and $\phi$ - then $\varepsilon_{j}^{\sigma} \ll|\Delta|$. Moreover, if $k$ is large enough - independent of $E_{1}$ and $\phi-$ then $|\Delta|<\varepsilon_{k}^{\sigma}$. So we assume these inequalities.

We shall also assume that $\alpha_{k}$ is continuous on $\Delta$. This can be achieved by choosing the sets $\Lambda_{l}\left(m_{l}\right), l<k$, appropriately, because if, by induction, $\alpha_{l}$ is continuous on $\Delta$, then

$$
\left|\alpha_{l}\left(\sqrt{E_{2}}\right)-\alpha_{l}\left(\sqrt{E_{1}}\right)\right| \leqq N_{l}^{\tau} \varepsilon_{l}^{-\sigma}|\Delta|<\varepsilon_{l}^{\sigma}
$$


This implies that the sets $\Lambda_{l}(m)$ can be chosen so that all points in $\Delta$ belongs to the same component. Hence $\alpha_{l+1}$ is continuous on $\Delta$.

Since $\left|2 \alpha_{k}\left(\sqrt{E_{1}}\right)-\left\langle m_{k}\right\rangle\right|<4 \varepsilon_{k}^{\sigma}$ and $2 \alpha_{k} \partial \alpha_{k}=\partial \operatorname{det} A_{k}$, it follows that the image of $\Delta$ under $\alpha_{k}$ is contained in an interval around $\frac{\left\langle m_{k}\right\rangle}{2}$ of diameter

$$
\leqq \varepsilon_{k}^{2 \sigma} N_{j}^{-4 \tau} N_{k}^{\tau} \varepsilon_{k}^{-\sigma}
$$

Hence, all $\sqrt{E} \in \Delta$ belongs to $\Lambda_{k}\left(m_{k}\right)$, and if $m \neq m_{j}$ and $\langle m\rangle \in 2 \alpha_{k}(\Delta)$, then $|m|>N_{j}$. In particular, $\alpha_{j}$ is continuous on $\Delta$. Finally, we get from corollary 6 that the set of all "gaps" in $\Delta$ is of measure

$$
\leqq 4 \varepsilon_{j}^{\sigma}+N_{j+1}^{\tau} 4 \varepsilon_{j+1}^{\sigma}+N_{j+2}^{\tau} 4 \varepsilon_{j+2}^{\sigma}+\cdots \leqq c \varepsilon_{j}^{\sigma} .
$$

Hence, if we let

$$
\Delta^{\prime}=\left\{\sqrt{E} \in \Delta: \sqrt{E} \in \bigcap_{l>k} \Lambda_{l}(0)\right\}
$$

then $\left|\Delta \backslash \Delta^{\prime}\right|<c \varepsilon_{j}^{\sigma}$. Moreover, on $\Delta^{\prime}$ we have that $|\alpha| \geqq \frac{1}{8} N_{j}^{-\tau}$, because otherwise we would have that $\left|\alpha_{j}(\sqrt{E})\right|<\frac{1}{4} N_{j}^{-\tau}$. But since $\left|\alpha_{j}\left(\sqrt{E_{1}}\right)\right| \geqq \frac{1}{2} N_{j}^{-\tau}$ and since $\alpha_{j}$ varies $\leqq \varepsilon_{k}^{2 \sigma} N_{j}^{-4 \tau} N_{j}^{3 \tau} \varepsilon_{k}^{-\sigma}<\frac{1}{4} N_{j}^{-\tau}$, this is impossible.

Let now

$$
\tilde{\Delta}=\{E: \sqrt{E} \in \Delta\}, \quad \tilde{\Delta}^{\prime}=\left\{E \in \tilde{\Delta}: \sqrt{E} \in \Delta^{\prime}\right\} .
$$

Then $\left|\tilde{\Delta} \backslash \tilde{\Delta}^{\prime}\right|<\frac{1}{2}|\tilde{\Delta}|$ so there must exist a $E_{2} \in \tilde{\Delta}^{\prime}$ such that

$$
r\left(E_{2}\right)|\tilde{\Delta}| \leqq 2 \int_{\tilde{\Delta}} r(s) d s .
$$

Now for $|g| \leqq|\tilde{\Delta}|$ we get by (8.2) that

$$
\int_{-\infty}^{+\infty} \frac{g}{\left(E_{2}-s\right)^{2}+g^{2}} d \mu(s)=r\left(E_{2}+i g\right) \leqq r\left(E_{2}\right)+1 .
$$

On the other hand we have for $|g|=|\tilde{\Delta}|$

$$
\begin{aligned}
r\left(E_{2}\right)+1 & \geqq \int_{-\infty}^{+\infty} \frac{g}{\left(E_{2}-s\right)^{2}+g^{2}} d \mu(s) \geqq \int_{\tilde{\Delta}} \frac{g}{\left(E_{2}-s\right)^{2}+g^{2}} d \mu(s) \\
& \geqq \frac{g}{g^{2}+g^{2}} d \mu(\tilde{\Delta})=\frac{1}{2|g|} d \mu(\tilde{\Delta}) .
\end{aligned}
$$

This implies that

$$
d \mu(\tilde{\Delta}) \leqq\left(r\left(E_{2}\right)+1\right) 2|g| \leqq 4 \int_{\tilde{\Delta}} r(\mu) d \mu+2|\tilde{\Delta}|,
$$

By choosing $k$ sufficiently large, we can get $\tilde{\Delta}$ arbitrarily small. This proves the lemma. 


\section{Appendix. On Rotation Number}

Let $X: \mathbf{R} \rightarrow G l(2, \mathbf{R})$ be continuous with $X(0)=I$. For any $V \in \mathbf{R}^{2} \backslash 0$ let

i.e.

$$
\phi(t, V)=-\arg (X(t) V),
$$

$$
\phi\left(t_{2}, V\right)-\phi\left(t_{1}, V\right)=-\operatorname{Im}\left(\int_{\gamma}^{1} \frac{1}{z} d z\right),
$$

where $\gamma$ is the curve $X(t) V, t_{1} \leqq t \leqq t_{2}$, in the complex plane. So $\phi$ is a continuous multi-valued function. We shall fix

$$
0 \leqq \arg (V)<2 \pi,
$$

so that $\phi$ becomes a single-valued function, continuous in $\mathbf{R} \times\left(\mathbf{R}^{2} \backslash([0, \infty[\times\{0\}))\right.$.

Suppose $\phi(t, V)-\phi(t, W)=n 2 \pi$. This implies that $X(t) V=a X(t) W$ for some $a>0$, i.e. $X(t)(V-a W)=0$. Hence $V=a W$. From this it follows that

$$
|\phi(t, V)-\phi(t, W)|<\pi
$$

for all $V, W \in \mathbf{R}^{2} \backslash 0$.

We say that $X$ has rotation number $\rho=\rho_{X}$ if

$$
\lim _{t \rightarrow \infty} \frac{\phi(t, V)}{t}=\rho
$$

for some, and hence for all, $V \in \mathbf{R}^{2} \backslash 0$. (A.1) implies that the convergence is uniform with respect to $V$.

If $X$ has rotation number $\rho$, then

$$
\lim _{t \rightarrow \infty} \frac{\left(\phi(t, V)-\phi\left(t_{0}, V\right)\right)}{t}=\rho,
$$

which shows that the rotation number does not depend on the values of $X$ over a finite interval.

So if $Y: \mathbf{R} \rightarrow G l(2, \mathbf{R})$ is continuous with $Y(0)=I$, then we have the following lemma.

Lemma A.1. Suppose $X(t)=Y(t)$ for $t \geqq T$. If $\rho_{X}$ exists, then $\rho_{Y}=\rho_{X}$.

Suppose now that $\rho_{X}$ and $\rho_{Y}$ exist. Let

$$
\psi(t, V)=-\arg (Y(t) V), \quad f(t, V)=-\arg (Y(t) X(t) V) .
$$

Then

$$
f(t, V)-f(0, V)=-\operatorname{Im}\left(\int_{\gamma}^{1} \frac{1}{z} d z\right)=-\operatorname{Im}\left(\int_{\gamma_{1}} \frac{1}{z} d z\right)-\operatorname{Im}\left(\int_{\gamma_{2}} \frac{1}{z} d z\right)
$$

where

$$
\begin{cases}\gamma=Y(s) X(s) V & 0 \leqq s \leqq t \\ \gamma_{1}=X(s) V & 0 \leqq s \leqq t \\ \gamma_{2}=Y(s) X(t) V & 0 \leqq s \leqq t\end{cases}
$$


Hence,

$$
f(t, V)-f(0, V)=(\phi(t, V)-\phi(0, V))+(\psi(t, X(t) V)-\psi(0, X(t) V)),
$$

which proves the following lemma.

Lemma A2. If $\rho_{X}$ and $\rho_{Y}$ exist, then $\rho_{Y X}=\rho_{x}+\rho_{Y}$.

Let now $A \in s l(2, \mathbf{R})$ with purely imaginary eigenvalues $\pm i \alpha$. Then there exists a real matrix $M$, det $M>0$, such that $M^{-1} A M=\alpha J$-this defines the sign of $\alpha$-and from this we see that $e^{A t}$ has rotation number $\alpha$. In fact $e^{A t}$ has the same rotation number as $X(t) e^{\alpha J t} X(t)^{-1}$, where $X(t)=M$ for $t \geqq 1$.

In the same way one shows that if $A$ has real eigenvalues then the rotation number of $e^{A t}$ is 0 .

Consider now an equation

$$
X^{\prime}(t)=F(t) X(t), \quad F=\left(\begin{array}{ll}
a & b \\
c & d
\end{array}\right),
$$

where $F$ is continuous, and let $\phi$ be a solution of

$$
-\phi^{\prime}(t)=(a(t)-d(t)) \cos \phi(t) \sin \phi(t)-(b(t)+c(t)) \cos ^{2} \phi(t)+b(t),
$$

with $\phi(0)=\phi_{0}$. Then

$$
\frac{d}{d t} e^{-\phi J}=F e^{-\phi J}-e^{-\phi J} T,
$$

where $T=T(t)$ is triangular. Hence, we have a solution $X(t)=e^{\phi(t) J} Y(t)$, where $Y$, being a solution of $Y^{\prime}=T Y$, can be taken to be triangular. From this it follows that $\rho_{X}$ exists if and only if

$$
\lim _{t \rightarrow \infty} \frac{\phi(t)}{t}
$$

exists, and that $\rho_{X}$ is precisely this limit. Moreover, if $F$ is $\mathrm{q}-\mathrm{p}$, then this limit does indeed exist as stated in the following lemma for whose proof we refer to [1].

Lemma A.3. Let $f: \mathbf{T}^{d+1} \rightarrow \mathbf{R}$ be continuous, and let $\omega \in \mathbf{R}$ be rationally independent. If

$$
\phi^{\prime}(t)=f(\omega t+x, \phi(t)), \quad \phi(0)=\phi_{0},
$$

then

$$
\lim _{t \rightarrow \infty} \frac{\phi(t)}{t}
$$

exists. Moreover, this limit is independent of $\phi_{0}$ and $x \in \mathbf{T}^{d}$.

So if $F$ is q-p, then $X$ has rotation number

$$
\lim _{t \rightarrow \infty} \frac{\phi(t)}{t}=-\lim _{t \rightarrow \infty} \frac{1}{t} \int_{0}^{t}(a(s)-d(s)) \cos \phi(s) \sin \phi(s)-(b(s)+c(s)) \cos ^{2} \phi(s)+b(s) d s
$$


which is bounded by

$$
\sup _{t \in \mathbb{R}}(|a(t)-d(t)|+\mid(b(t)+c(t)|+| b(t) \mid
$$

In particular, if $F_{n}$ goes uniformly to 0 as $n \rightarrow \infty$, then $\rho_{X_{n}} \rightarrow 0$ as well.

Acknowledgements. Most of this work was done in 1989 during a stay at the Forschungsinstitut für Mathematic at ETH in Zürich for whose hospitality. I am very grateful. I also like to thank J. Moser for the interest he showed in this work.

\section{References}

1. Johnson, R., Moser, J.: The rotation number for almost periodic potentials. Commun. Math. Phys. 84, 403-438 (1982)

2. Moser, J., Pöschel, J.: An extension of a result by Dinaburg and Sinai on quasi-periodic potentials. Comment. Math. Helvetici 59, 39-85 (1984)

3. Dinaburg, E. I., Sinai, Y. G.: The one dimensional Schrödinger equation with quasi-periodic potential. Funkt. Anal. i. Priloz. 9, 8-21 (1975)

4. Rüssmann, H.: On the one dimensional Schrödinger equation with a quasi-periodic potential. Ann. N. Y. Acad. Sci. 357, 90-107 (1980)

5. Deift, P., Simon, B.: Almost periodic Schrödinger operators. III. The absolutely continuous spectrum in one dimension. Commun. Math. Phys. 90, 389-411 (1983)

6. Fröhlich, J., Spencer, T., Wittwer, P.: Localization for a class of one dimensional quasi-periodic Schrödinger operators. Commun. Math. Phys. 132, 5-25 (1990)

7. Sinai, Ya. G.: Anderson localization for the one-dimensional difference Schrödinger operator with quasiperiodic potentials. J. Stat. Phys. 46, 861-909 (1987)

8. Surace, S.: The Schrödinger equation with a quasi-periodic potential, Thesis N. Y. University

9. Spencer, T.: Ergodic Schrodinger operators. In P. Rabinoqitz, E. Zehnder (eds.). Analysis etc. Volume for J. Moser's sixtieth birthday, New York Press 1989

10. Kotani, S.: Lyapunov Indices determine absolutely continuous spectra of stationary random 1-dimensional Schrödinger operators. Proc. of Taniguchi Sympos., SA Katata, 225-247, (1982)

11. Chulaevsky, V. A., Delyon, F.: Purely absolutely continuous spectrum for almost Mathieu operators, preprint (1989)

12. Albanese, C.: Quasiperiodic Schrödinger operators with pure absolutely continuous spectrum, preprint Courant Institute, New York (1990)

13. Jörgens, K., Rellich, F.: Eigenwerttheorie gewöhnlicher Differentialgleichungen. Berlin, Heidelberg, New York: 1976

14. Dym, H., McKean, H. P.: Gaussian Processes, Function Theory, and the Inverse spectral Problem. New York: Academic Press (1972)

Communicated by Ya. G. Sinai 